\title{
Joint UAV Hovering Altitude and Power Control for Space-Air-Ground IoT Networks
}

\author{
Jingjing Wang, Student Member, IEEE, Chunxiao Jiang, Senior Member, IEEE, \\ Zhongxiang Wei, Member, IEEE, Cunhua Pan, Member, IEEE \\ Haijun Zhang, Senior Member, IEEE and Yong Ren, Senior Member, IEEE
}

\begin{abstract}
Unmanned aerial vehicles (UAVs) have been widely used in both military and civilian applications. Equipped with diverse communication payloads, UAVs cooperating with satellites and base stations (BSs) constitute a space-air-ground three-tier heterogeneous network, which are beneficial in terms of both providing the seamless coverage as well as of improving the capacity for increasingly prosperous Internet of Things (IoT) networks. However, cross-tier interference may be inevitable among these tightly embraced heterogeneous networks when sharing the same spectrum. The power association problem in satellite, UAV and macrocell three-tier networks becomes a critical issue. In our paper, we propose a two-stage joint hovering altitude and power control solution for the resource allocation problem in UAV networks considering the inevitable cross-tier interference from space-air-ground heterogeneous networks. Furthermore, Lagrange dual decomposition and concaveconvex procedure (CCP) method are used to solve this problem, followed by a low-complexity greedy search algorithm. Finally, simulation results show the effectiveness of our proposed twostage joint optimization algorithm in terms of UAV network's total throughput.
\end{abstract}

Index Terms-UAV communication networks, IoT network, power control, cross-tier interference, heterogeneous networks, satellite.

\section{INTRODUCTION}

This work is supported by the National Natural Science Foundation of China (91338203, 61822104, 61471025, 61771044), the Young Elite Scientist Sponsorship Program by CAST (2016QNRC001), the Pre-research fund of Equipments of Ministry of Education of China (6141A02022615), the Co-Research with the fifth Research Institute of China Aerospace Science and Technology Corporation (Co-20180605-47), the Research Foundation of Ministry of Education of China \& China Mobile (MCM20170108), Beijing Natural Science Foundation (L172025, L172049), 111 Project (No. B170003), and the Fundamental Research Funds for the Central Universities (FRF-GF17-A6, RC1631). (Corresponding author: Chunxiao Jiang)

J. Wang and Y. Ren are with the Department of Electronic Engineering, Tsinghua University, Beijing, 100084, China. E-mail: chinaeephd@gmail.com, reny@tsinghua.edu.cn.

C. Jiang is with Tsinghua Space Center, Tsinghua University, Beijing, 100084, China. E-mail: jchx@tsinghua.edu.cn.

Z. Wei is with the University of Liverpool, Liverpool L69 3BX, U.K. Email: hszwei@liv.ac.uk.

C. Pan is with the Queen Mary University of London, London E1 4NS, U.K. E-mail: c.pan@qmul.ac.uk.

H. Zhang is with Beijing Advanced Innovation Center for Materials Genome Engineering, Beijing Engineering and Technology Research Center for Convergence Networks and Ubiquitous Services, Institute of Artificial Intelligence, University of Science and Technology Beijing, Beijing, 100083, China. E-mail: haijunzhang@ieee.org.

Copyright (c) 2012 IEEE. Personal use of this material is permitted However, permission to use this material for any other purposes must be obtained from the IEEE by sending a request to pubs-permissions@ieee.org.
G IVEN the substantial success of unmanned aerial vehicles (UAVs) in surveillance and monitoring tasks, it has become vitally important to bring drones into wireless communications considering their low cost, fast deployment, fully controllable mobility as well as the line of sight (LOS) communication links. These drones are usually equipped with diverse payloads for receiving, processing and transiting signals, which can be viewed as the aerial mobile base station (AMBS) constituting UAV communication networks [1] [2].

UAV communication networks along with traditional satellite networks and ground cellulars construct a space-air-ground three-tier heterogeneous network, which is capable of both providing seamless coverage as well as of further improving the channel capacity for increasingly prosperous Internet of Things (IoT) networks [3]-[5]. More explicitly, the ground marcocell base station (MBS) provides the basic broad-band information services for the IoT nodes. Small drones act as on-demand aerial access points for the sake of offloading the ground MBS and of constructing emergency links in the context of contingency. Moreover, UAV netowrk can be viewed as a promising solution to support energy-efficient uplink and location in energy-constrained IoT-centric networks [6]. By contrast, the satellite is used for providing a global coverage benefitting from its broadcast services and broad sight [7]. Hence, relying on such three-tier heterogeneous network, the connectivity, capacity and energy efficiency of IoT networks can be significantly improved.

In particular, as a beneficial communication enhancement facilitator, UAV network is characterized by flexility, costsaving and energy-efficiency. Specifically, in remote regions not seamlessly covered by macrocells on the ground, the UAV network may economically provide information services for IoT nodes compared with the high cost of satellite connections. Furthermore, in crowded places the UAV network may relieve the channel congestion of the macrocell and guarantee the quality of service (QoS) of latency- and throughput-sensitive IoT applications [8] [9]. More importantly, UAVs can help to quickly construct an emergency information system or even act as the IoT sensing nodes, which is beneficial of supporting the disaster relief when a large part of cellular and Internet infrastructures on the ground are destroyed by a calamity [10].

UAV aided hybrid communication techniques have been widely investigated in the literature. Specifically, in [11], Amorim et al. presented models for path loss exponents and shadowing of the radio channel between UAVs and cellular networks with the aid of a field measurement. Zeng et al. 
proposed an energy-efficient UAV communication model by optimizing the trajectory of drones, which jointly considered both the energy consumption and the communication throughput in [12]. The energy efficiency of UAV communication was defined as the ratio of total transmitted bits to UAV's energy consumption. Furthermore, an effective dynamic trajectory control mechanism for multi-UAV network was proposed by Fadlullah et al. in [13], which was beneficial in terms of both improving the network throughput as well as of reducing the communication delay. In [14], Mozaffari et al. considered a coexistence between the UAVs and an underlaid device-to-device (D2D) communication network and studied the coverage performance as well as data rate. However, the aforementioned articles mostly focused their attention on how to improve the communication performance such as throughput, delay and coverage, by designing the mobility of drones, while few considered the intra- and inter-interference and cross-tier resource allocation among different networks.

Due to spectrum scarcity, it is possible to sharing the spectrum among different kinds of communication subsystems. More specifically, the C-band, Ku-band and Ka-band have been well utilized for air-to-ground reliable wide-band communications. Particularly, a range of compelling applications of the fifth generation wireless systems (5G) attempt to use higher frequency band for providing low-latency and highthroughput services, such as C-band and Ka-band, which are originally assigned to the airborne communications [15]-[18]. It is worth noting that mmWave communications [19] have been already adopted in both UAV and satellite scenarios [20][23]. Hence, a well-implemented network association mechanism of space-air-ground heterogeneous systems is beneficial in terms of both improving the resource utilization as well as of reducing the cross-tier interference [24] [25] [26]. As for the resource allocation problem in heterogeneous networks, Fooladivanda et al. in [27] investigated the user association and resource allocation in heterogeneous cellular networks in terms of orthogonal channel deployment, co-channel deployment as well as partially shared channel deployment. Moreover, they obtained upper bounds of the heterogeneous network's performance. Furthermore, a distributed joint allocation algorithm is proposed for band selection and power allocation in order to maximize total capacity of a multi-mode and multi-band user terminal (MMT) by Choi et al. in [28]. Moreover, considering the aspect of energy efficiency, Xie et al. in [29] formulated an energy-efficient resource allocation problem as a Stackelberg game for heterogeneous cognitive femtocells, followed by a gradient based iteration solution. In [30], Ye et al. focused their attention on the relationship between the user association and load balancing for heterogeneous networks with picocells and femtocells solved by a low-complexity and fast-convergence distributed algorithm. Furthermore, in [31], a mixed-integer programming problem was formulated for allocating subchannel and power resources in orthogonal frequency-division multiple access (OFDMA) hybrid networks with femtocells.

However, these resource allocation mechanisms may not be suitable for the applications for the UAV aided spaceair-ground heterogeneous network, because few of them considered the characteristics of UAVs in designing resource allocation algorithms, such as dynamic topology, flexible deployment, etc. Given a general space-air-ground heterogeneous communication scenario jointly served by the satellite, lowaltitude UAVs and the ground MBS, users served by UAV networks may severely influence or be inevitably affected by the operation of satellite communication systems and macrocells. Therefore, the resource allocation of different kinds of users should take into account the inevitable crosstier interference in space-air-ground hybrid networks [32]. Moreover, as the aerial base stations, UAVs play a critical role in offloading the ground MBS and in enhancing ultra-reliable communication links. Given the coverage of each drone network, frequently changing UAVs' horizontal hovering position in the same altitude may result in server inter-interference between adjacent UAV networks and increase the risk of flight collision. Additionally, considering the power constraint of small drones, it may be unrealistic to make the drone adaptively move around for supporting the bursty traffic of the ground users. Hence, a delicately designed UAVs' hovering altitude distribution is capable of improving the user's QoS by deploying more drones in different hovering altitudes as well as of guaranteeing their flight safety.

Inspired by the above-mentioned open challenges, in this paper, we propose a two-stage joint hovering altitude and power control for UAV networks in the context of a spaceair-ground heterogeneous communication network considering diverse user's QoS requirements. Our main contributions can be summarized as follows:

- To the best of our knowledge, this is the first work for resource allocation for UAV networks in the context of space-air-ground heterogeneous communication systems considering the feasible deployment of drones aiming to reduce the cross-tier interference.

- A two-stage joint hovering altitude and power control solution is investigated for our proposed model. Both the Lagrange dual decomposition and concave-convex procedure (CCP) method are used to approximatively solve the relaxed convex problem. Moreover, a low-complexity greedy proportionable power constrained algorithm is proposed for resource allocation in UAV networks.

- Extensive simulations are conducted in order to evaluate the performance of our proposed two-stage joint resource allocation scheme. Simulation results show that our resource allocation mechanism is beneficial in terms of improving UAV network's total throughput considering the inevitable cross-tier interference.

The remainder of this article is outlined as follows. The system model and problem formulation are detailed in Section II. A two-stage joint hovering altitude and power control solution for UAV networks is elaborated in Section III. In Section IV, the implementation of the aforementioned two-stage joint iterative algorithm as well as a low-complexity greedy algorithm is presented, including its complexity analysis. In Section V, simulation results are provided for characterizing our proposed uplink resource allocation model for UAV networks, followed by our conclusions in Section VI. 


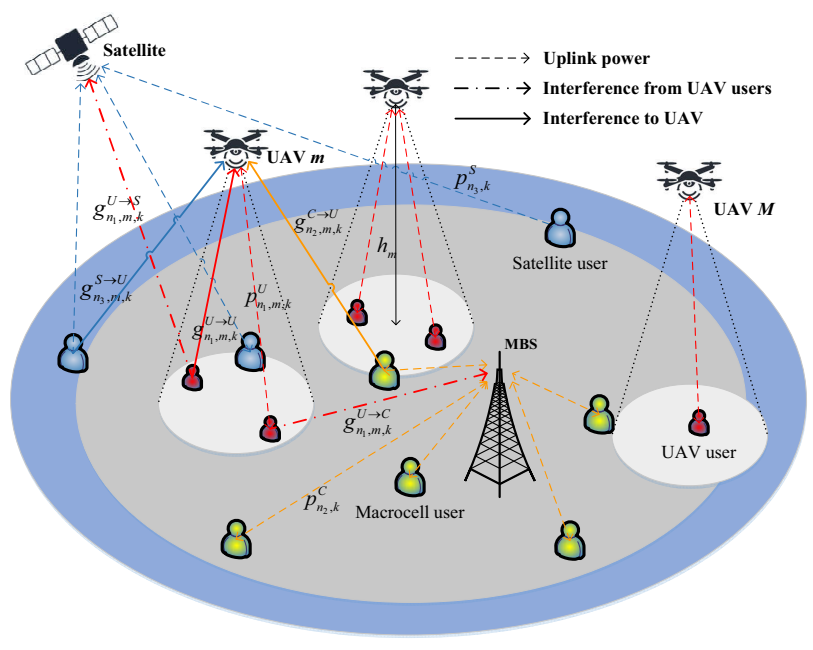

Fig. 1. The structure of satellite, UAV and macrocell three-tier hybrid network.

\section{System Model AND PRoblem Formulation}

\section{A. System Model}

In this paper, as show in Fig. 1, we consider a three-tier hybrid network including a satellite network with a geosynchronous earth orbit satellite (GEO), a macrocell with a MBS and $M$ UAV networks sharing the same channel. Each UAV network is served by a hovering drone. Let $h_{m}$ represent the hovering altitude of the $m$-th drone. The coverage of $M \mathrm{UAV}$ networks are overlaid within the coverage of the GEO as well as the macrocell. We focus our attention on the uplink power control of the users in the UAV networks. We assume that the uplink power of both satellite users and of macrocell users is equal.

The bandwidth of the channel is $B$, which is divided into $K$ subchannels. The channel fading between the MBS and users on the ground is the frequency-selective Reyleigh fading, while the communication channel between the hovering drone and users is dominated by the line of sight $(\mathrm{LoS})$ path. The channel fading between the GEO and users on the ground is the Rice fading.

Let $N_{S}$ and $N_{C}$ denote the number of active users served by the GEO and by the MBS in a macrocell, respectively. Moreover, $N_{U}$ is the number of active users camping on each UAV network. We assume that the satellite users and the macrocell users are uniformly distributed in each coverage area. In our model, two kinds of users with different QoS requirements are served in each UAV network. Specifically, the number of QoS-sensitive users requiring a high transmission rate of $R_{h}$ is $N_{u h}$, while the number of QoS-tolerant users with a low transmission rate requirement of $R_{l}$ is $N_{u l}$, where $N_{u h}+N_{u l}=N_{U}$. Let $\mathbb{N}_{u h}$ and $\mathbb{N}_{u l}$ represent the set of QoSsensitive users and QoS-tolerant users, respectively. Then, we have $\left|\mathbb{N}_{u h}\right|=N_{u h}$ and $\left|\mathbb{N}_{u l}\right|=N_{u l}$, and $\mathbb{N}_{u h} \bigcap \mathbb{N}_{u l}=\emptyset$.

Let $g_{n_{1}, m, k}^{U \rightarrow S}, g_{n_{1}, m, k}^{U \rightarrow C}$ and $g_{n_{1}, m, k}^{U \rightarrow U}$ denote the channel gains on $k$-th subchannel from user $n_{1}$ in $m$-th UAV network to the GEO, to the MBS and to the hovering drone, respectively, where $n_{1} \in\left\{1,2, \ldots, N_{U}\right\}, m \in\{1,2, \ldots, M\}$ and $k \in\{1,2, \ldots, K\}$. In our model, $g_{n_{1}, m, k}^{U \rightarrow S}$ can be viewed as a constant because the UAV users locate far away from the GEO satellite, while $g_{n_{1}, m, k}^{U \rightarrow C}$ depends the channel state and the distance between each UAV user and the MBS. For the sake of analysis, we assume that the service radius of each drone can be neglected compared with its altitude, and hence $g_{n_{1}, m, k}^{U \rightarrow U}$ is only sensitive to the hovering altitude $h_{m}$ of the $m$-th drone, which can be formulated as:

$$
g_{n_{1}, m, k}^{U \rightarrow U}=\frac{\kappa}{h_{m}^{2}},
$$

where $\kappa$ denotes the unit power gain in terms of the reference distance $h_{r}=1 \mathrm{~m}$. Furthermore, let $g_{n_{2}, m, k}^{C \rightarrow U}$ represent the channel gain on $k$-th subchannel from user $n_{2}$ in the macrocell to the $m$-th hovering drone, while $g_{n_{3}, m, k}^{S \rightarrow U}$ denotes the channel gain on $k$-th subchannel from user $n_{3}$ in the satellite network to the $m$-th hovering drone, where $n_{2} \in\left\{1,2, \ldots, N_{C}\right\}$ and $n_{3} \in\left\{1,2, \ldots, N_{S}\right\}$. Moreover, let $p_{n_{2}, k}^{C}$ and $p_{n_{3}, k}^{S}$ represent the uplink transmission power of user $n_{2}$ in the macrocell and of user $n_{3}$ in the satellite network on $k$-th subchannel, respectively, while $p_{n_{1}, m, k}^{U}$ is the uplink transmission power of user $n_{1}$ in the $m$-th UAV network on $k$-th subchannel. In our model, we define $\mathbf{P}_{N_{U} \times M \times K}$ as the power allocation matrix for the users served by total $M$ UAV networks, and we have $[\mathbf{P}]_{n_{1}, m, k}=p_{n_{1}, m, k}^{U}$.

Here, we define a channel indicator matrix as $\mathbf{A}_{N_{U} \times M \times K}$, where $[\mathbf{A}]_{n_{1}, m, k}=a_{n_{1}, m, k}$. To elaborate, $a_{n_{1}, m, k}=1$ represents that the $k$-th subchannel is occupied by user $n_{1}$ in the $m$-UAV network, otherwise, $a_{n_{1}, m, k}=0$. We consider the additive white Gaussian noise (AWGN) with the variance of $\sigma^{2}$. Hence, as for the $m$-th UAV network, the received signalto-interference-plus-noise ratio (SINR) of the hovering drone from user $n_{1}$ accessing the $k$-th subchannel can be calculated by:

$$
\gamma_{n_{1}, m, k}=\frac{p_{n_{1}, m, k}^{U} g_{n_{1}, m, k}^{U \rightarrow U}}{g_{n_{2}, m, k}^{C \rightarrow U} p_{n_{2}, k}^{C}+g_{n_{3}, m, k}^{S \rightarrow U} p_{n_{3}, k}^{S}+\sigma^{2}},
$$

where $g_{n_{2}, m, k}^{C \rightarrow U} p_{n_{2}, k}^{C}$ is the interference from the user in the macrocell sharing the same sub-channel, while $g_{n_{3}, m, k}^{S \rightarrow U} p_{n_{3}, k}^{S}$ is the interference caused by the user in the satellite network occupying the $k$-th sub-channel. Remarkably, at most one user is capable of accessing the same subchannel at one moment in the macrocell, in the satellite network as well as in a UAV network. For the sake of simplification, in our model we assume that the users served by the drones are equipped with a directional antenna and the co-interference between different UAV networks is negligible compared with the crosstier interference from the macrocell and the satellite network.

Relying on the Shannon formula [33], the uplink capacity of $m$-th UAV network from its user $n_{1}$ on $k$-th subchannel can be calculated by:

$$
C_{n_{1}, m, k}=\frac{B}{K} \log _{2}\left(1+\gamma_{n_{1}, m, k}\right) .
$$

\section{B. Problem Formulation}

In this section, we will formulate the uplink resource allocation problem for the UAV network. Furthermore, we 
assume that the channel state information (CSI) as well as the result of uplink resource allocation can be forwarded to the users by the hovering drone based on the channel reciprocity.

1) Constraints: In our uplink resource allocation problem, our objective is to maximize the total capacity in $M$ UAV networks under the following constraints:

- UAV user's power constraint: the users in each UAV network have a maximum transmission power limit of $P_{\max }^{U}$. Hence, for $\forall n_{1} \in\left\{1,2, \ldots, N_{U}\right\}$ and $\forall m \in$ $\{1,2, \ldots, M\}$, we have:

$$
\sum_{k=1}^{K} a_{n_{1}, m, k} p_{n_{1}, m, k}^{U} \leq P_{\max }^{U} .
$$

Furthermore, the non-negativity of power yields $p_{n_{1}, m, k}^{U} \geq 0$.

- UAV safety flight and hovering altitude constraint: In order to guarantee the safety of $M$ cooperated drones, we consider a hierarchical deployment of these drones with different hovering altitudes. Moreover, the hovering altitudes of them are distributed within a specified safe range of $\left[h_{\min }, h_{\max }\right]$ and subjects to:

$$
\sum_{i, j \in \mathbb{M}, i \neq j}\left(h_{i}-h_{j}\right)^{2} \geq \chi^{2},
$$

where $\chi^{2}$ is the minimal variance of the altitude of $M$ drones for safety flight and hovering, while $\mathbb{M}$ represents the set of $M$ hovering drones. Moreover, for $\forall i, j \in \mathbb{M}$, we have $h_{\min } \leq h_{i}, h_{j} \leq h_{\max }$.

- QoS guarantee: For the QoS-sensitive users, the requirement of a high transmission rate of $R_{h}$ can be expressed as:

$$
\sum_{k=1}^{K} a_{n_{u h}, m, k} C_{n_{u h}, m, k} \geq R_{h},
$$

where $\forall n_{u h} \in \mathbb{N}_{u h}$ and $\forall m \in\{1,2, \ldots, M\}$. By contrast, for the QoS-tolerant users, we have:

$$
\sum_{k=1}^{K} a_{n_{u l}, m, k} C_{n_{u l}, m, k} \geq R_{l}
$$

where $\forall n_{u l} \in \mathbb{N}_{u l}$ and $\forall m \in\{1,2, \ldots, M\}$.

- Interference constraint of macrocell: UAV networks share the same frequency with the macrocell. Hence, the macrocell may suffer a cross-tier interference from $M$ UAV networks. Let $I_{k}^{C}$ denote the threshold of the interference on the $k$-th subchannel and $\forall k \in\{1,2, \ldots, K\}$, i.e.

$$
\sum_{m=1}^{M} \sum_{n_{1}=1}^{N_{U}} a_{n_{1}, m, k} p_{n_{1}, m, k}^{U} g_{n_{1}, m, k}^{U \rightarrow C} \leq I_{k}^{C} .
$$

- Interference constraint of satellite network: Similar to the interference constraint of macrocell, let $I_{k}^{S}$ represent the threshold of the interference from UAV networks to the satellite network on the $k$-th subchannel. Thus, we have:

$$
\sum_{m=1}^{M} \sum_{n_{1}=1}^{N_{U}} a_{n_{1}, m, k} p_{n_{1}, m, k}^{U} g_{n_{1}, m, k}^{U \rightarrow S} \leq I_{k}^{S},
$$

where $\forall k \in\{1,2, \ldots, K\}$.
- Subchannel allocation constraint: In each UAV network, one subchannel can be allocated to at most one user, which can be formulated by:

$$
\sum_{n_{1}=1}^{N_{U}} a_{n_{1}, m, k} \leq 1
$$

where $\forall k \in\{1,2, \ldots, K\}$ and $\forall m \in\{1,2, \ldots, M\}$. Moreover, the channel indicator variable $a_{n_{1}, m, k} \in$ $\{0,1\}$.

2) Uplink Resource Allocation Formulation: The total capacity of $M$ UAV networks can be given by:

$$
C_{\text {total }}=\sum_{m=1}^{M} \sum_{n_{1}=1}^{N_{U}} \sum_{k=1}^{K} a_{n_{1}, m, k} C_{n_{1}, m, k} .
$$

Hence, the uplink resource allocation problem can be formulated as:

$$
\begin{aligned}
& \max _{\left\{a_{n_{1}, m, k}, p_{n_{1}, m, k}^{U}, h_{m}\right\}} \sum_{m=1}^{M} \sum_{n_{1}=1}^{N_{U}} \sum_{k=1}^{K} a_{n_{1}, m, k} C_{n_{1}, m, k} \\
& \text { s.t. } \quad(12 \mathrm{a}): \sum_{k=1}^{K} a_{n_{1}, m, k} p_{n_{1}, m, k}^{U} \leq P_{\max }^{U}, \quad \forall n_{1}, m \text {, } \\
& \text { (12b): } p_{n_{1}, m, k}^{U} \geq 0, \quad \forall n_{1}, m, k, \\
& \text { (12c) : } \sum_{i, j \in \mathbb{M}, i \neq j}\left(h_{i}-h_{j}\right)^{2} \geq \chi^{2} \text {, } \\
& (12 \mathrm{~d}): h_{\min } \leq h_{m} \leq h_{\max }, \quad \forall m \\
& \text { (12e) : } \sum_{k=1}^{K} a_{n_{u h}, m, k} C_{n_{u h}, m, k} \geq R_{h}, \quad \forall n_{u h}, m, \\
& \text { (12f) : } \sum_{k=1}^{K} a_{n_{u l}, m, k} C_{n_{u l}, m, k} \geq R_{l}, \quad \forall n_{u l}, m, \\
& (12 \mathrm{~g}): \sum_{m=1}^{M} \sum_{n_{1}=1}^{N_{U}} a_{n_{1}, m, k} p_{n_{1}, m, k}^{U} g_{n_{1}, m, k}^{U \rightarrow C} \leq I_{k}^{C}, \quad \forall k, \\
& \text { (12h) : } \sum_{m=1}^{M} \sum_{n_{1}=1}^{N_{U}} a_{n_{1}, m, k} p_{n_{1}, m, k}^{U} g_{n_{1}, m, k}^{U \rightarrow S} \leq I_{k}^{S}, \quad \forall k \text {, } \\
& \text { (12i) : } \sum_{n_{1}=1}^{N_{U}} a_{n_{1}, m, k} \leq 1, \quad \forall m, k, \\
& (12 \mathrm{j}): a_{n_{1}, m, k} \in\{0,1\}, \quad \forall n_{1}, m, k \text {. }
\end{aligned}
$$

To elaborate further, (12a) and (12b) in problem (12) are users' power constraints, while (12c) and (12d) are hovering altitude constraints. As for the QoS constraints (12e) and (12f), considering the QoS-tolerant users with a low transmission rate requirement of $R_{l}$, where $0<R_{l} \ll R_{h}$, hence we can neglect the constraint (12f) in problem (12) without loss of generality. Furthermore, $(12 \mathrm{~g})$ and $(12 \mathrm{~h})$ are interference constraint from macrocell and satellite network, respectively. Finally, the subchannel allocation constraints are given by (12i) and $(12 \mathrm{j})$. Unfortunately, however, our optimization objective is a function of $\left(a_{n_{1}, m, k}, p_{n_{1}, m, k}^{U}, h_{m}\right)$, and the form of $a_{n_{1}, m, k} C_{n_{1}, m, k}$ is not concave in $\left(a_{n_{1}, m, k}, p_{n_{1}, m, k}^{U}, h_{m}\right)$. Moreover, the hovering altitude constraint of (12c) and the 
integer programming constraint of $(12 \mathrm{j})$ are not convex as well. In the following, we will reformulate the optimization problem (12) as a convex optimization problem with the aid of relaxing the integer constraints and provide its solution relying on a two-stage joint optimization.

\section{Two-Stage Joint Hovering Altitude And Power CONTROL SOLUTION}

In this section, we propose a two-stage joint optimization algorithm for our uplink resource allocation problem. Since there are a total of three kinds of optimization variables in problem (12), i.e $a_{n_{1}, m, k}, p_{n_{1}, m, k}^{U}$ and $h_{m}$, it is prohibitive to find the globally optimal solution and thus a near optimal algorithm with low computational complexity is desirable. In the following, we first fix the hovering altitude $h_{m}=h_{m}^{0}, m \in$ $\mathbb{M}$ and search the optimal joint subchannel and power control scheme in Stage 1. Then, relying on the result of Stage 1, we try to find the optimal hovering altitude of each drone in Stage 2 .

\section{A. Stage 1: Joint Subchannel and Power Control}

1) Constraint Relaxation: Here, we first study the joint subchannel and power control problem with given hovering altitude, where the initial $h_{m}^{0}$ constitutes a arithmetic progression ranging from $h_{\min }$ to $h_{\max }$. In the following, we convert the non-convex problem (12) into a tractable convex problem [31] [34] [35]. First of all, we relax the inter programming constraint $a_{n_{1}, m, k} \in\{0,1\}$ in (12j) to a continuous convex constraint $a_{n_{1}, m, k} \in[0,1]$.

Furthermore, let us introduce the auxiliary variable $\rho_{n_{1}, m, k}=a_{n_{1}, m, k} p_{n_{1}, m, k}^{U}$, and hence the uplink capacity of Eq. (3) can be converted to:

$$
\begin{aligned}
& \hat{C}_{n_{1}, m, k}= \\
& \frac{B}{K} \log _{2}\left(1+\frac{\rho_{n_{1}, m, k} g_{n_{1}, m, k}^{U \rightarrow U}}{a_{n_{1}, m, k}\left(g_{n_{2}, m, k}^{C \rightarrow U} p_{n_{2}, k}^{C}+g_{n_{3}, m, k}^{S \rightarrow U} p_{n_{3}, k}^{S}+\sigma^{2}\right)}\right),
\end{aligned}
$$

where $g_{n_{1}, m, k}^{U \rightarrow U}=\frac{\kappa}{h_{m}^{2}}$ and $h_{m} \triangleq h_{m}^{0}, m \in \mathbb{M}$. Now we introduce Lemma 1 to show the concavity of our objective function.

Lemma 1. Assume $f(x)$ is a concave function of $x$ when $x \in[0, X]$. Let us introduce a variable a as $a=t x, t \in[0,1]$. Then, $g(t, a)=t f(a / t)$ is concave in $(t, a)$ when $t \in[0,1]$ and $\forall a \in[0, t X]$.

Proof. Since $f(x)$ is a concave function, $f^{\prime \prime}(x) \leq 0$. The Hessian matrix of $g(t, a)$ can be calculated as:

$$
\nabla^{2} g(t, a)=\frac{f^{\prime \prime}(a / t)}{t^{3}}\left[\begin{array}{cc}
a^{2} & -a t \\
-a t & t^{2}
\end{array}\right]
$$

Furthermore, for $\forall x, y \in \mathbb{R}$ and $t \in[0,1]$, we have:

$$
\left[\begin{array}{ll}
x & y
\end{array}\right] \nabla^{2} g(t, a)\left[\begin{array}{l}
x \\
y
\end{array}\right]=\frac{f^{\prime \prime}(a / t)}{t^{3}}(a x-y t)^{2} \leq 0 .
$$

Hence, the Hessian matrix $\nabla^{2} g(t, a)$ is a negative semidefinite matrix. Thus, $g(t, a)=t f(a / t)$ is a concave function [36] [37].
Relying on Lemma 1, our optimization objective $a_{n_{1}, m, k} \hat{C}_{n_{1}, m, k}$ is concave in $\left(a_{n_{1}, m, k}, \rho_{n_{1}, m, k}\right)$, based on which our joint subchannel and power control problem can be reformulated as:

$$
\begin{aligned}
& \max _{\left\{a_{n_{1}, m, k}, \rho_{n_{1}, m, k}\right\}} \sum_{m=1}^{M} \sum_{n_{1}=1}^{N_{U}} \sum_{k=1}^{K} a_{n_{1}, m, k} \hat{C}_{n_{1}, m, k} \\
& \text { s.t. } \quad(14 \mathrm{a}): \sum_{k=1}^{K} \rho_{n_{1}, m, k} \leq P_{\max }^{U}, \quad \forall n_{1}, m \text {, } \\
& \text { (14b) : } \rho_{n_{1}, m, k} \geq 0, \quad \forall n_{1}, m, k, \\
& \text { (14c) : } \sum_{k=1}^{K} a_{n_{u h}, m, k} \hat{C}_{n_{u h}, m, k} \geq R_{h}, \quad \forall n_{u h}, m \text {, } \\
& \text { (14d) : } \sum_{m=1}^{M} \sum_{n_{1}=1}^{N_{U}} \rho_{n_{1}, m, k} g_{n_{1}, m, k}^{U \rightarrow C} \leq I_{k}^{C}, \quad \forall k \\
& \text { (14e) : } \sum_{m=1}^{M} \sum_{n_{1}=1}^{N_{U}} \rho_{n_{1}, m, k} g_{n_{1}, m, k}^{U \rightarrow S} \leq I_{k}^{S}, \quad \forall k, \\
& \text { (14f) : } \sum_{n_{1}=1}^{N_{U}} a_{n_{1}, m, k} \leq 1, \quad \forall m, k, \\
& (14 \mathrm{~g}): a_{n_{1}, m, k} \in[0,1], \quad \forall n_{1}, m, k \text {. }
\end{aligned}
$$

Obviously, our joint subchannel and power control problem in (14) is a convex optimization problem.

2) Lagrangian Dual Decomposition Method: In this subsection, we use the Lagrangian dual decomposition method to solve our joint subchannel and power control problem in (14) [38]. Let $L(\mathbf{A}, \boldsymbol{\rho}, \boldsymbol{\lambda}, \boldsymbol{\mu}, \boldsymbol{\nu}, \boldsymbol{\omega}, \boldsymbol{\xi})$ be the Lagrangian function, which can be written as:

$$
\begin{aligned}
L & (\mathbf{A}, \boldsymbol{\rho}, \boldsymbol{\lambda}, \boldsymbol{\mu}, \boldsymbol{\nu}, \boldsymbol{\omega}, \boldsymbol{\xi}) \\
& =\sum_{m=1}^{M} \sum_{n_{1}=1}^{N_{U}} \sum_{k=1}^{K} a_{n_{1}, m, k} \hat{C}_{n_{1}, m, k} \\
& +\sum_{m=1}^{M} \sum_{n_{1}=1}^{N_{U}} \lambda_{n_{1}, m}\left(P_{\max }^{U}-\sum_{k=1}^{K} \rho_{n_{1}, m, k}\right) \\
& +\sum_{m=1}^{M} \sum_{n_{u h}=1}^{N_{u h}} \mu_{n_{u h}, m}\left(\sum_{k=1}^{K} a_{n_{u h}, m, k} \hat{C}_{n_{u h}, m, k}-R_{h}\right) \\
& +\sum_{k=1}^{K} \nu_{k}\left(I_{k}^{C}-\sum_{m=1}^{M} \sum_{n_{1}=1}^{N_{U}} \rho_{n_{1}, m, k} g_{n_{1}, m, k}^{U \rightarrow C}\right) \\
& +\sum_{k=1}^{K} \omega_{k}\left(I_{k}^{S}-\sum_{m=1}^{M} \sum_{n_{1}=1}^{N_{U}} \rho_{n_{1}, m, k} g_{n_{1}, m, k}^{U \rightarrow S}\right) \\
& +\sum_{m=1}^{M} \sum_{k=1}^{K} \xi_{m, k}\left(1-\sum_{n_{1}=1}^{N_{U}} a_{n_{1}, m, k}\right),
\end{aligned}
$$

where $\boldsymbol{\lambda}, \boldsymbol{\mu}, \boldsymbol{\nu}, \boldsymbol{\omega}$ and $\boldsymbol{\xi}$ are the Lagrange multipliers associated with the corresponding constraints, while $\mathbf{A}=\left\{a_{n_{1}, m, k}\right\}$ and $\boldsymbol{\rho}=\left\{\rho_{n_{1}, m, k}\right\}$. The constraints of $(14 \mathrm{~b})$ and (14g) will be considered after obtaining the optimal solution of $a_{n_{1}, m, k}$ $\rho_{n_{1}, m, k}$. 
Hence, the Lagrangian dual function can be expressed as:

$$
g(\boldsymbol{\lambda}, \boldsymbol{\mu}, \boldsymbol{\nu}, \boldsymbol{\omega}, \boldsymbol{\xi})=\sup _{\mathbf{A}, \boldsymbol{\rho}} L(\mathbf{A}, \boldsymbol{\rho}, \boldsymbol{\lambda}, \boldsymbol{\mu}, \boldsymbol{\nu}, \boldsymbol{\omega}, \boldsymbol{\xi}) .
$$

The Lagrangian dual problem can be formulated as:

$$
\begin{gathered}
\min _{\boldsymbol{\lambda}, \boldsymbol{\mu}, \boldsymbol{\nu}, \boldsymbol{\omega}, \boldsymbol{\xi}} g(\boldsymbol{\lambda}, \boldsymbol{\mu}, \boldsymbol{\nu}, \boldsymbol{\omega}, \boldsymbol{\xi}) \\
\text { s.t. } \quad \boldsymbol{\lambda}, \boldsymbol{\mu}, \boldsymbol{\nu}, \boldsymbol{\omega}, \boldsymbol{\xi} \succeq 0 .
\end{gathered}
$$

Eq. (15) can be reorganized as:

$$
L(\mathbf{A}, \boldsymbol{\rho}, \boldsymbol{\lambda}, \boldsymbol{\mu}, \boldsymbol{\nu}, \boldsymbol{\omega}, \boldsymbol{\xi})=\sum_{m=1}^{M} \sum_{k=1}^{K} \boldsymbol{\Phi}+\boldsymbol{\Psi}
$$

where

$$
\begin{aligned}
& \boldsymbol{\Phi}=\sum_{n_{1}=1}^{N_{U}} a_{n_{1}, m, k} \hat{C}_{n_{1}, m, k}-\sum_{n_{1}=1}^{N_{U}} \lambda_{n_{1}, m} \rho_{n_{1}, m, k} \\
& +\sum_{n_{u h}=1}^{N_{u h}} \mu_{n_{u h}, m} a_{n_{u h}, m, k} \hat{C}_{n_{u h}}-\sum_{n_{1}=1}^{N_{U}} \nu_{k} \rho_{n_{1}, m, k} g_{n_{1}, m, k}^{U \rightarrow C} \\
& -\sum_{n_{1}=1}^{N_{U}} \omega_{k} \rho_{n_{1}, m, k} g_{n_{1}, m, k}^{U \rightarrow S}-\sum_{n_{1}=1}^{N_{U}} \xi_{m, k} a_{n_{1}, m, k},
\end{aligned}
$$

and

$$
\begin{aligned}
\Psi & =\sum_{m=1}^{M} \sum_{n_{1}=1}^{N_{U}} \lambda_{n_{1}, m} P_{\max }^{U}-\sum_{m=1}^{M} \sum_{n_{u h}=1}^{N_{u h}} \mu_{n_{u h}, m} R_{h} \\
& +\sum_{k=1}^{K} \nu_{k} I_{k}^{C}+\sum_{k=1}^{K} \omega_{k} I_{k}^{S}+\sum_{m=1}^{M} \sum_{k=1}^{K} \xi_{m, k} .
\end{aligned}
$$

Relying on Eq. (18), the dual problem can be divided into $(M \times K)$ parallel subproblems. Let $a_{n_{1}, m, k}^{*}$ and $\rho_{n_{1}, m, k}^{*}$ represent the optimal solutions of maximizing the Eq. (19). Take the partial derivative of Eq. (19) with respect to $a_{n_{1}, m, k}$ and $\rho_{n_{1}, m, k}$, and for the QoS-sensitive user $i \in \mathbb{N}_{u h}$, we have:

$$
\frac{\partial \Phi}{\partial \rho_{i, m, k}}=\frac{B}{K \ln 2}\left(\frac{a_{i, m, k} g_{i, m, k}^{U \rightarrow U}+\mu_{i, m} a_{i, m, k} g_{i, m, k}^{U \rightarrow U}}{a_{i, m, k} \Delta+\rho_{i, m, k}^{*} g_{n_{1}, m, k}^{U \rightarrow U}}\right)-\Theta_{i},
$$

while for the QoS-tolerant user $j \in \mathbb{N}_{u l}$, i.e.

$$
\frac{\partial \boldsymbol{\Phi}}{\partial \rho_{j, m, k}}=\frac{B}{K \ln 2}\left(\frac{a_{j, m, k} g_{j, m, k}^{U \rightarrow U}}{a_{j, m, k} \Delta+\rho_{j, m, k}^{*} g_{j, m, k}^{U \rightarrow U}}\right)-\Theta_{j}
$$

where $\Delta=g_{n_{2}, m, k}^{C \rightarrow U} p_{n_{2}, k}^{C}+g_{n_{3}, m, k}^{S \rightarrow U} p_{n_{3}, k}^{S}+\sigma^{2}$ and $\Theta_{n_{1}}=$ $\lambda_{n_{1}, m}+\nu_{k} g_{n_{1}, m, k}^{U \rightarrow C}+\omega_{k} g_{n_{1}, m, k}^{U \rightarrow S}, n_{1} \in\left\{1,2, \ldots, N_{U}\right\}$. Considering the constraint of (14b), as $\boldsymbol{\Phi}$ is also a concave function, the optimal solution $\rho_{n_{1}, m, k}^{*}, \forall n_{1}, m, k$ obeys:

$$
\left\{\begin{array}{l}
\rho_{n_{1}, m, k}^{*}=0 \quad \text { and }\left.\quad \frac{\partial \boldsymbol{\Phi}}{\partial \rho_{n_{1}, m, k}}\right|_{\rho_{n_{1}, m, k}=0}<0, \\
\rho_{n_{1}, m, k}^{*}>0 \quad \text { and }\left.\quad \frac{\partial \boldsymbol{\Phi}}{\partial \rho_{n_{1}, m, k}}\right|_{\rho_{n_{1}, m, k}=\rho_{n_{1}, m, k}^{*}}=0 .
\end{array}\right.
$$

Then, the optimal solution of the power allocation $p_{n_{1}, m, k}^{U *}=$ $\rho_{n_{1}, m, k}^{*} / a_{n_{1}, m, k}$ in $m$-th UAV network on the $k$-th subchannel for user $n_{1}$ can be given by:

$p_{n_{1}, m, k}^{U *}=\left\{\begin{array}{l}\max \left\{0, \frac{B\left(1+\mu_{i, m}\right)}{K \ln 2 \times \Theta_{n_{1}}}-\frac{\Delta}{g_{j, m, k}^{U \rightarrow U}}\right\}, n_{1} \in \mathbb{N}_{u h}, \\ \max \left\{0, \frac{B}{K \ln 2 \times \Theta_{n_{1}}}-\frac{\Delta}{g_{j, m, k}^{U \rightarrow U}}\right\}, n_{1} \in \mathbb{N}_{u l} .\end{array}\right.$

Similarly, considering $a_{n_{1}, m, k}^{*} \in[0,1]$ in $(14 \mathrm{~g})$, the optimal solution $a_{n_{1}, m, k}^{*}, \forall n_{1}, m, k$ are given by:

$$
\left\{\begin{array}{l}
a_{n_{1}, m, k}^{*}=0 \quad \text { and }\left.\quad \frac{\partial \boldsymbol{\Phi}}{\partial a_{n_{1}, m, k}}\right|_{a_{n_{1}, m, k}=0}<0, \\
a_{n_{1}, m, k}^{*} \in(0,1) \quad \text { and }\left.\quad \frac{\partial \boldsymbol{\Phi}}{\partial a_{n_{1}, m, k}}\right|_{a_{n_{1}, m, k}=a_{n_{1}, m, k}^{*}}=0 \\
a_{n_{1}, m, k}^{*}=1 \quad \text { and }\left.\quad \frac{\partial \boldsymbol{\Phi}}{\partial a_{n_{1}, m, k}}\right|_{a_{n_{1}, m, k}=1}>0
\end{array}\right.
$$

where for the QoS-sensitive user $i \in \mathbb{N}_{u h}$,

$$
\begin{aligned}
& \frac{\partial \boldsymbol{\Phi}}{\partial a_{i, m, k}}=\left(1+\mu_{i, m}\right) \frac{B}{K} \log _{2}\left(1+\frac{p_{i, m, k}^{U *} g_{i, m, k}^{U \rightarrow U}}{\Delta}\right) \\
& -\left(1+\mu_{i, m}\right) \frac{B p_{i, m, k}^{U *} g_{i, m, k}^{U \rightarrow U}}{K \ln 2 \times\left(\Delta+p_{i, m, k}^{U *} g_{i, m, k}^{U \rightarrow U}\right)}-\lambda_{i, m} p_{i, m, k}^{U *} \\
& -\nu_{k} p_{i, m, k}^{U *} g_{i, m, k}^{U \rightarrow C}-\omega_{k} p_{i, m, k}^{U *} g_{i, m, k}^{U \rightarrow S}-\xi_{m, k},
\end{aligned}
$$

and for the QoS-tolerant user $j \in \mathbb{N}_{u l}$, we have:

$$
\begin{aligned}
\frac{\partial \boldsymbol{\Phi}}{\partial a_{j, m, k}} & =\frac{B}{K} \log _{2}\left(1+\frac{p_{j, m, k}^{U *} g_{j, m, k}^{U \rightarrow U}}{\Delta}\right) \\
& -\frac{B p_{j, m, k}^{U *} g_{j, m, k}^{U \rightarrow U}}{K \ln 2 \times\left(\Delta+p_{j, m, k}^{U *} g_{j, m, k}^{U \rightarrow U}\right)}-\lambda_{j, m} p_{j, m, k}^{U *} \\
& -\nu_{k} p_{j, m, k}^{U *} g_{j, m, k}^{U \rightarrow C}-\omega_{k} p_{j, m, k}^{U *} g_{j, m, k}^{U \rightarrow S}-\xi_{m, k} .
\end{aligned}
$$

In our model, at most one user is allowed to access the same subchannel at one moment in a UAV network. In order to maximize the Lagrangian function, we have:

$$
n_{1}^{*}=\arg \max _{n_{1}} \frac{\partial \Phi}{\partial a_{n_{1}, m, k}}, \quad \forall m, k,
$$

where $a_{n_{1}^{*}, m, k}^{*}=1$ represents the suboptimal channel indicator variable.

3) The Update of Lagrangian Multipliers: Since the Lagrangian dual function in Eq. (16) is not differentiable, we use the subgradient method to update the Lagrangian multipliers $\boldsymbol{\lambda}, \boldsymbol{\mu}, \boldsymbol{\nu}, \boldsymbol{\omega}$ and $\boldsymbol{\xi}$ [39] [40] [41]. The Lagrangian multipliers can be updated as follows:

$$
\lambda_{n_{1}, m}^{(i+1)}=\left[\lambda_{n_{1}, m}^{(i)}-\alpha_{1}^{(i)}\left(P_{\max }^{U}-\sum_{k=1}^{K} \rho_{n_{1}, m, k}\right)\right]^{+}, \forall m, n_{1},
$$

$\mu_{n_{u h}, m}^{(i+1)}=\left[\mu_{n_{u h}, m}^{(i)}-\alpha_{2}^{(i)}\left(\sum_{k=1}^{K} a_{n_{u h}, m, k} \hat{C}_{n_{u h}, m, k}-R_{h}\right)\right]^{+}, \forall m, n_{u h}$,

$\nu_{k}^{(i+1)}=\left[\nu_{k}^{(i)}-\alpha_{3}^{(i)}\left(I_{k}^{C}-\sum_{m=1}^{M} \sum_{n_{1}=1}^{N_{U}} \rho_{n_{1}, m, k} g_{n_{1}, m, k}^{U \rightarrow C}\right)\right]^{+}, \forall k$, 


$$
\omega_{k}^{(i+1)}=\left[\omega_{k}^{(i)}-\alpha_{4}^{(i)}\left(I_{k}^{S}-\sum_{m=1}^{M} \sum_{n_{1}=1}^{N_{U}} \rho_{n_{1}, m, k} g_{n_{1}, m, k}^{U \rightarrow S}\right)\right]^{+}, \forall k,
$$

where $i$ is the indicator of the iteration, and $\alpha$ represents the step size, while $[\cdot]^{+}=\max \{0, \cdot\}$. Moreover, to guarantee the convergence of the subgradient method, the step sizes should satisfy:

$$
\sum_{i=1}^{\infty} \alpha^{(i)}=\infty, \quad \text { and } \quad \lim _{i \rightarrow \infty} \alpha^{(i)}=0 .
$$

In order to speed up the convergence, an adaptive step size is set as $\alpha=1 / I$, where $I$ represents the iteration index. Relying on Eq. (24)-Eq. (33), we can obtain the optimal solution $\left\{a_{n_{1}^{*}, m, k}^{*}, p_{n_{1}, m, k}^{U *}\right\}$ of joint subchannel and power control for each users in UAV networks considering a fixed deployment altitude of hovering drones. Denote the obtained total capacity of UAV networks as $C_{\text {total }}\left(a_{n_{1}^{*}, m, k}^{*}, p_{n_{1}, m, k}^{U *}, \mathbf{h}\right)$, where $\mathbf{h} \triangleq\left[h_{1}, h_{2}, \ldots, h_{M}\right]^{T}$.

\section{B. Stage 2: Hovering Altitude Optimization}

1) Difference of Convex Programming Based Hovering Altitude Formulation: As mentioned before, in Stage 1, we fix the deployment altitude of each hovering drone and search for the optimal joint subchannel and power control mechanism for each user in UAV network, denoted as $\left\{a_{n_{1}^{*}, m, k}^{*}, p_{n_{1}, m, k}^{U *}\right\}$, where $n_{1} \in\left\{1,2, \ldots, N_{U}\right\}, m \in\{1,2, \ldots, M\}$ and $k \in$ $\{1,2, \ldots, K\}$. In the following, we try to determine the optimal hovering altitude of each drone based on the results obtained from Stage 1. Considering the safety hovering altitude constraints of $(12 \mathrm{c})$ and $(12 \mathrm{~d})$ in our original problem formulation in (12), we have:

$$
\begin{array}{ll}
\max _{\left\{h_{m}\right\}} & \sum_{m=1}^{M} \sum_{n_{1}=1}^{N_{U}} \sum_{k=1}^{K} a_{n_{1}^{*}, m, k}^{*} \frac{B}{K} \log _{2}\left(1+\frac{\kappa p_{n_{1}, m, k}^{U *}}{h_{m}^{2} \Delta}\right) \\
\text { s.t. } & (34 \mathrm{a}): \sum_{i, j \in \mathbb{M}, i \neq j}\left(h_{i}-h_{j}\right)^{2} \geq \chi^{2}, \\
& (34 \mathrm{~b}): h_{m} \leq h_{\max }, \quad \forall m, \\
& (34 \mathrm{c}): h_{m} \geq h_{\min }, \quad \forall m .
\end{array}
$$

The hovering altitude optimization problem in (34) can be reformulated as a difference of convex (DC) programming [42] [43], which can be given by:

$$
\begin{array}{ll}
\min _{\mathbf{h}} \quad 0-g_{0}(\mathbf{h}) & \\
\text { s.t. } & (35 \mathrm{a}): \chi^{2}-g_{1}(\mathbf{h}) \leq 0, \\
& (35 \mathrm{~b}): h_{m} \leq h_{\max }, \quad \forall m, \\
& (35 \mathrm{c}): h_{m} \geq h_{\min }, \quad \forall m .
\end{array}
$$

where the objective function can be expressed as:

$$
g_{0}(\mathbf{h})=\sum_{m=1}^{M} \sum_{n_{1}=1}^{N_{U}} \sum_{k=1}^{K} a_{n_{1}^{*}, m, k}^{*} \frac{B}{K} \log _{2}\left(1+\frac{\kappa p_{n_{1}, m, k}^{U *}}{h_{m}^{2} \Delta}\right),
$$

and $g_{1}(\mathbf{h})$ can be given by:

$$
g_{1}(\mathbf{h})=\sum_{i, j \in \mathbb{M}, i \neq j}\left(h_{i}-h_{j}\right)^{2} .
$$

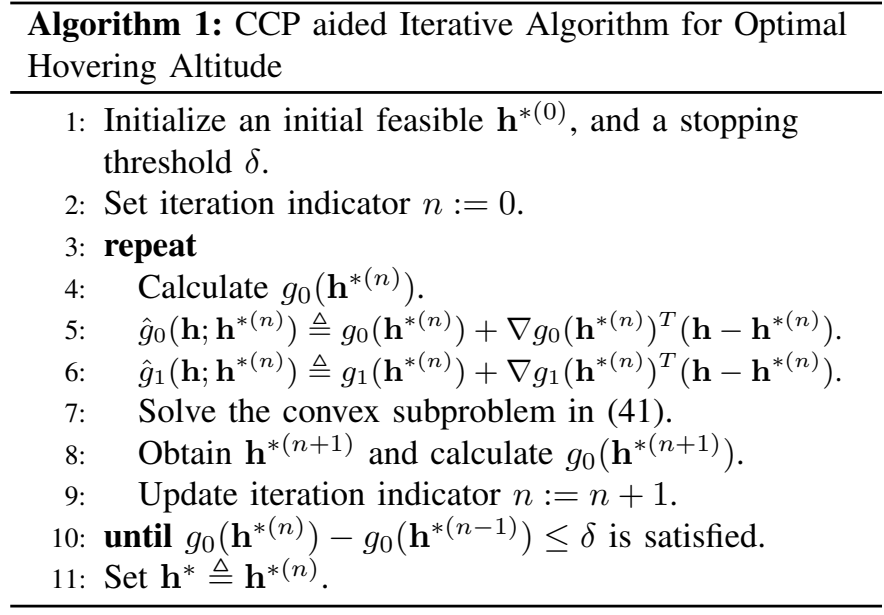

Specifically, $g_{1}(\mathbf{h})$ is a quadratic form, which can be rewritten as $g_{1}(\mathbf{h})=\mathbf{h}^{T} \mathbf{Q h}$, where $\mathbf{Q}=\operatorname{diag}(M)-\mathbf{1}$. Moreover, $\operatorname{diag}(M)$ denotes a diagonal matrix with all diagonal elements equaling $M$ and $\mathbf{1}$ is an $M \times M$ matrix with all elements being 1. Hence, both $g_{0}(\mathbf{h})$ and $g_{1}(\mathbf{h})$ in (35) are convex functions. Hence, we can use the CCP method to solve the problem in (35), where we are capable of achieving the locally optimal result of the non-convex problem through solving a series of iterative convex subproblems as shown in Algorithm. 1.

2) CCP Aided Iterative Solation: To elaborate a litter further, let $\hat{g}_{0}(\mathbf{h})$ and $\hat{g}_{1}(\mathbf{h})$ approximatively be the first-order Taylor expansion of $g_{0}(\mathbf{h})$ and $g_{1}(\mathbf{h})$, respectively, i.e.

$$
\begin{aligned}
\hat{g}_{0}\left(\mathbf{h} ; \mathbf{h}^{(n)}\right) & \triangleq g_{0}\left(\mathbf{h}^{(n)}\right)+\nabla g_{0}\left(\mathbf{h}^{(n)}\right)^{T}\left(\mathbf{h}-\mathbf{h}^{(n)}\right) \\
& =g_{0}\left(\mathbf{h}^{(n)}\right)+\Gamma\left(\mathbf{h}^{(n)}\right)^{T}\left(\mathbf{h}-\mathbf{h}^{(n)}\right),
\end{aligned}
$$

and

$$
\begin{aligned}
\hat{g}_{1}\left(\mathbf{h} ; \mathbf{h}^{(n)}\right) & \triangleq g_{1}\left(\mathbf{h}^{(n)}\right)+\nabla g_{1}\left(\mathbf{h}^{(n)}\right)^{T}\left(\mathbf{h}-\mathbf{h}^{(n)}\right) \\
& =\mathbf{h}^{(n) T} \mathbf{Q} \mathbf{h}^{(\mathbf{n})}+\left(2 \mathbf{Q} \mathbf{h}^{(n)}\right)^{T}\left(\mathbf{h}-\mathbf{h}^{(n)}\right),
\end{aligned}
$$

where $\mathbf{h}^{(n)}$ is the value of $\mathbf{h}$ in the $n$-th iteration. Moreover, the $M \times 1$ vector $\Gamma=\frac{\mathrm{d} g_{0}(\mathbf{h})}{\mathrm{dh}}$, and the $m$-th element of $\Gamma$ can be calculated as:

$$
\Gamma_{m}=-\sum_{n_{1}=1}^{N_{U}} \sum_{k=1}^{K} \frac{2 \kappa B a_{n_{1}^{*}, m, k}^{*} p_{n_{1}, m, k}^{U *}}{K \ln 2 \times\left(h_{m}^{3} \Delta+h_{m} \kappa p_{n_{1}, m, k}^{U *}\right)} .
$$

Thus, the value of $\mathbf{h}^{(n+1)}$ can be achieved from solving the following series of convex linear-constraint subproblems:

$$
\begin{aligned}
& \min _{\mathbf{h}} 0-\hat{g}_{0}(\mathbf{h}) \\
& \text { s.t. }(41 \mathrm{a}): \chi^{2}-\hat{g}_{1}\left(\mathbf{h} ; \mathbf{h}^{(n)}\right) \leq 0 .
\end{aligned}
$$

Note that, the constrains of (35b) and (35c) in problem (35) will be considered in solving above-mentioned convex subproblem. The stopping criterion of the iteration can be given by:

$$
g_{0}\left(\mathbf{h}^{(n+1)}\right)-g_{0}\left(\mathbf{h}^{(n)}\right) \leq \delta,
$$

where $\delta$ is the stopping threshold. 
Here, we also use the Lagrangian dual decomposition method to solve the convex problem (41). The Lagrangian function can be given by:

$$
L(\mathbf{h}, \psi)=-\hat{g}_{0}(\mathbf{h})+\psi\left(\chi^{2}-\hat{g}_{1}(\mathbf{h})\right),
$$

where $\psi$ is the Lagrangian multiplier. Hence, the Lagrangian duality function is denoted as:

$$
z(\psi)=\inf _{\mathbf{h}}-\hat{g}_{0}(\mathbf{h})+\psi\left(\chi^{2}-\hat{g}_{1}(\mathbf{h})\right) .
$$

Then, the Lagrangian duality problem can be formulated as:

$$
\begin{aligned}
& \max _{\psi} z(\psi) \\
& \text { s.t. } \psi \geq 0 .
\end{aligned}
$$

Take the derivative of Eq. (51) against hovering altitude vector $\mathbf{h}$, and we have:

$$
\frac{\mathrm{d} L(\mathbf{h}, \psi)}{\mathrm{d} \mathbf{h}}=-\Gamma\left(\mathbf{h}^{(n)}\right)-2 \psi \mathbf{Q} \mathbf{h}^{(n)} .
$$

For $\forall m=\{1,2, \ldots, M\}$, we have:

$$
\begin{aligned}
& \frac{\mathrm{d} L\left(h_{m}\right)}{\mathrm{d} h_{m}}=2 \psi\left(\sum_{i=1, i \neq m}^{M} h_{i}^{(n)}-(M-1) h_{m}^{(n)}\right) \\
& +\sum_{n_{1}=1}^{N_{U}} \sum_{k=1}^{K} \frac{2 \kappa B a_{n_{1}^{*}, m, k}^{*} p_{n_{1}, m, k}^{U *}}{K \ln 2 \times\left(h_{m}^{3(n)} \Delta+h_{m}^{(n)} \kappa p_{n_{1}, m, k}^{U *}\right)},
\end{aligned}
$$

where $h_{m}^{(n)}$ is the value of $h_{m}$ in the $n$-th iteration. Hence, considering the fact that $L\left(h_{m}\right)$ is a convex function, we have:

$$
h_{m}^{(n+1)}= \begin{cases}h_{\min }, & \text { if } \frac{\mathrm{d} L\left(h_{m}\right)}{\mathrm{d} h_{m}}>0, \\ h_{\max }, & \text { if } \frac{\mathrm{d} L\left(h_{m}\right)}{\mathrm{d} h_{m}}<0 .\end{cases}
$$

Given that the dual function $z(\psi)$ is not differentiable, the Lagrangian multiplier $\psi$ can be updated by:

$$
\psi^{(n+1)}=\left[\psi^{(n)}+\beta^{(n)}\left(\chi^{2}-\hat{g}_{1}\left(\mathbf{h}^{n}\right)\right]^{+},\right.
$$

where $\beta^{(n)}$ is the step size of Lagrangian multiplier.

Then, we can achieve the optimal $h_{(n+1)}$ for the convex subproblem in (41). Hence, relying on the CCP aided iterative algorithm, given fixed $\left\{a_{n_{1}^{*}, m, k}^{*}, p_{n_{1}, m, k}^{U *}\right\}$, we obtain the optimal hovering altitude vector represented by $\mathbf{h}^{*}$. Moreover, for the sake of reducing computational complexity, numerous Boolean optimization algorithms can also be invoked in order to solve the near optimum of $\mathbf{h}$. Thus, the total capacity of UAV networks can be recalculated as $C_{\text {total }}\left(a_{n_{1}^{*}, m, k}^{*}, p_{n_{1}, m, k}^{U *}, \mathbf{h}^{*}\right)$. However, the pseudo-optimal capacity $C_{\text {total }}\left(a_{n_{1}^{*}, m, k}^{*}, p_{n_{1}, m, k}^{U *}, \mathbf{h}^{*}\right)$ is not the final optimal capacity of our proposed uplink resource allocation problem in (12), namely $C_{\text {total }}^{*}$. In the following, we will combine the aforementioned two stages in order to search for the optimal network capacity jointly considering the hovering altitude and the subchannel and power control ${ }^{1}$.

\footnotetext{
${ }^{1}$ Hereafter, we use $C_{\text {total }}^{(i)}\left(a_{n_{1}^{*}, m, k}^{*}, p_{n_{1}, m, k}^{U *}, \mathbf{h}^{*}\right)$ to represent the pseudooptimal capacity of the $i$-th two-stage iterative joint resource association for a more clear expression.
}

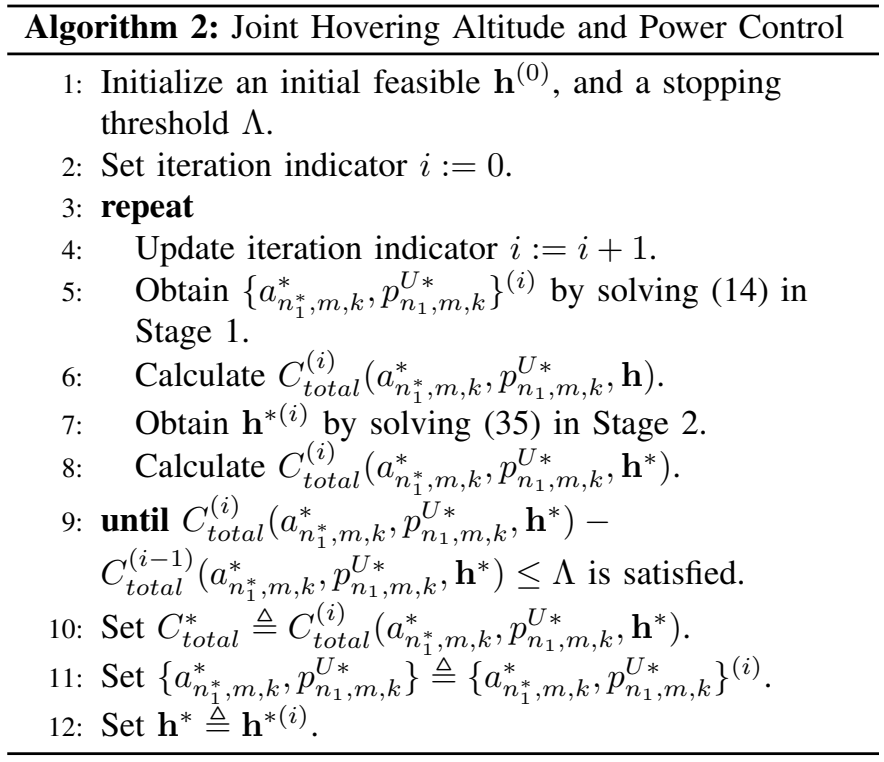

\section{Joint Hovering Altitude and Power Control}

1) Two-Stage Joint Resource Allocation: In Section III-A and Section III-B, we have studied the optimization problem of both the power control (Stage 1) and the hovering altitude (Stage 2). In this subsection, we combine these two stages and jointly optimize the hovering altitude and power control scheme. Specifically, in the $i$-th iteration, the optimal subchannel and power control of $\left\{a_{n_{1}^{*}, m, k}^{*}, p_{n_{1}, m, k}^{U *}\right\}^{(i)}$ can be achieved in Stage 1 based on fixed $\mathbf{h}^{(i-1)}$, yielding the total capacity of UAV networks of $C_{\text {total }}^{(i)}\left(a_{n_{1}^{*}, m, k}^{*}, p_{n_{1}, m, k}^{U *}, \mathbf{h}\right)$. Then, relying on given subchannel and power control of $\left\{a_{n_{1}^{*}, m, k}^{*}, p_{n_{1}, m, k}^{U *}\right\}^{(i)}$, in Stage 2, we can obtain the optimal hovering altitude vector $\mathbf{h}^{*(i)}$ and the pseudo-optimal capacity $C_{\text {total }}^{(i)}\left(a_{n_{1}^{*}, m, k}^{*}, p_{n_{1}, m, k}^{U *}, \mathbf{h}^{*}\right)$ in the $i$-th iteration of the two-stage joint resource allocation algorithm. In return, we conduct the optimization in Stage 1 based on the latest $\mathbf{h}^{*(i)}$ and update the $\left\{a_{n_{1}^{*}, m, k}^{*}, p_{n_{1}, m, k}^{U *}\right\}^{(i+1)}$ as well as $C_{\text {total }}^{(i+1)}\left(a_{n_{1}^{*}, m, k}^{*}, p_{n_{1}, m, k}^{U *}, \mathbf{h}\right)$. Then, relying on Stage 2, we can obtain the $\mathbf{h}^{*(i+1)}$ and the pseudo-optimal capacity $C_{\text {total }}^{(i+1)}\left(a_{n_{1}^{*}, m, k}^{*}, p_{n_{1}, m, k}^{U *}, \mathbf{h}^{*}\right)$.

Let $\Lambda$ be the stopping threshold of our two-stage resource allocation scheme. If the following condition is satisfied:

$C_{\text {total }}^{(i+1)}\left(a_{n_{1}^{*}, m, k}^{*}, p_{n_{1}, m, k}^{U *}, \mathbf{h}^{*}\right)-C_{\text {total }}^{(i)}\left(a_{n_{1}^{*}, m, k}^{*}, p_{n_{1}, m, k}^{U *}, \mathbf{h}^{*}\right) \leq \Lambda$,

the final optimal uplink total capacity of $M$ UAV networks can be given by:

$$
C_{\text {total }}^{*} \triangleq C_{\text {total }}^{(i+1)}\left(a_{n_{1}^{*}, m, k}^{*}, p_{n_{1}, m, k}^{U *}, \mathbf{h}^{*}\right),
$$

where the optimal subchannel and power control result is given by $\left\{a_{n_{1}^{*}, m, k}^{*}, p_{n_{1}, m, k}^{U *}\right\} \triangleq\left\{a_{n_{1}^{*}, m, k}^{*}, p_{n_{1}, m, k}^{U *}\right\}^{(i+1)}$ as well as the optimal hovering altitude $\mathbf{h}^{*} \triangleq \mathbf{h}^{*(i+1)}$. The procedure of the two-stage joint hovering altitude and power control optimization scheme for UAV networks is summarized in Algorithm. 2. 


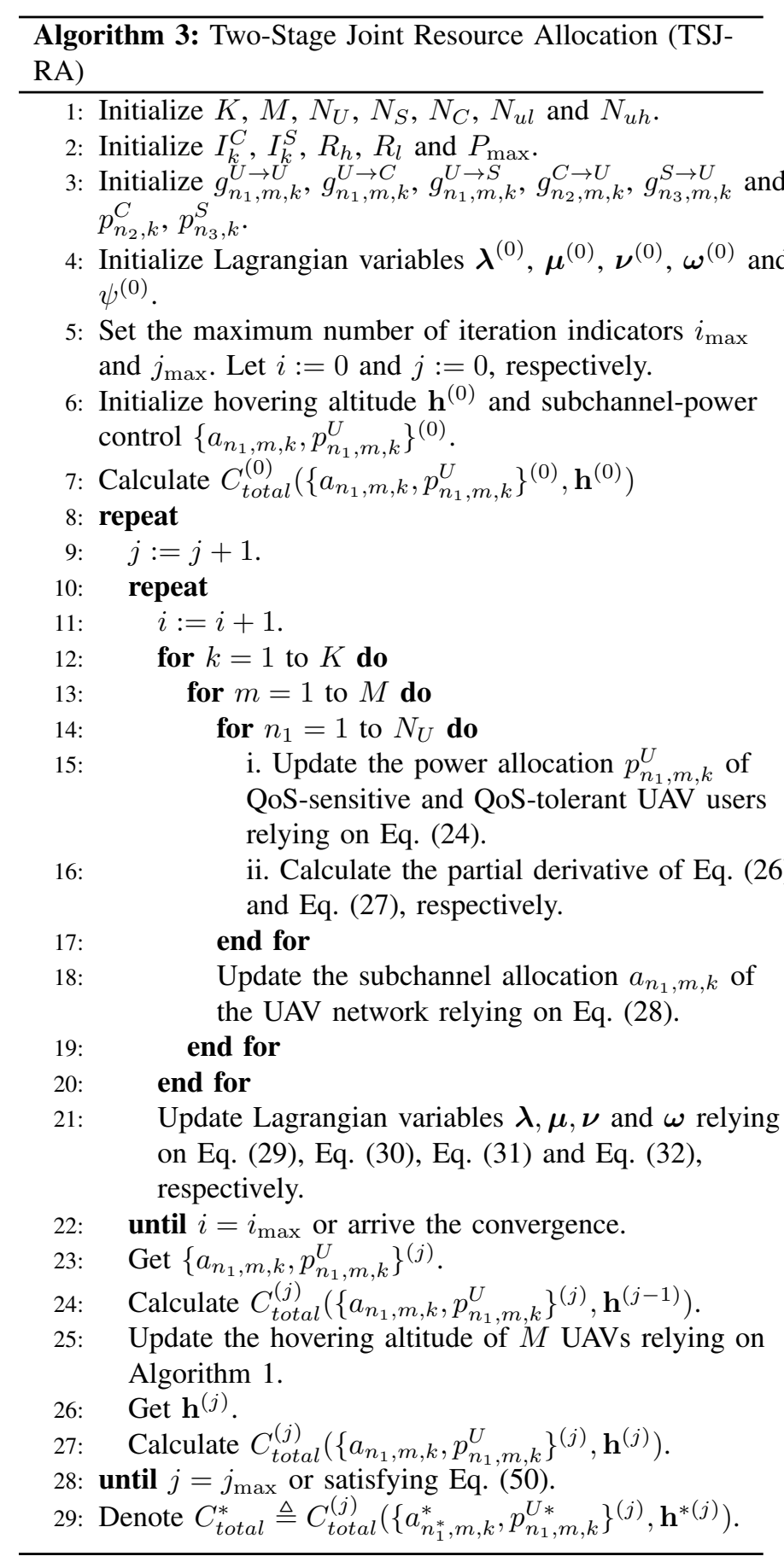

\section{Algorithm AND ANALYsis}

\section{A. Algorithm Implementation}

In this section, we will elaborate more on the algorithm implementation of our proposed UAV hovering altitude aided resource allocation mechanism described in Section III, namely TSJ-RA as shown in Algorithm 3 for space-air-ground three-tier heterogenous networks. Furthermore, to reduce the computational complexity we propose a heuristic resource allocation algorithm in Algorithm 4, i.e PPC-RA, which has lower computational complexity compared with the exhaustive search algorithm as well as with TSJ-RA.

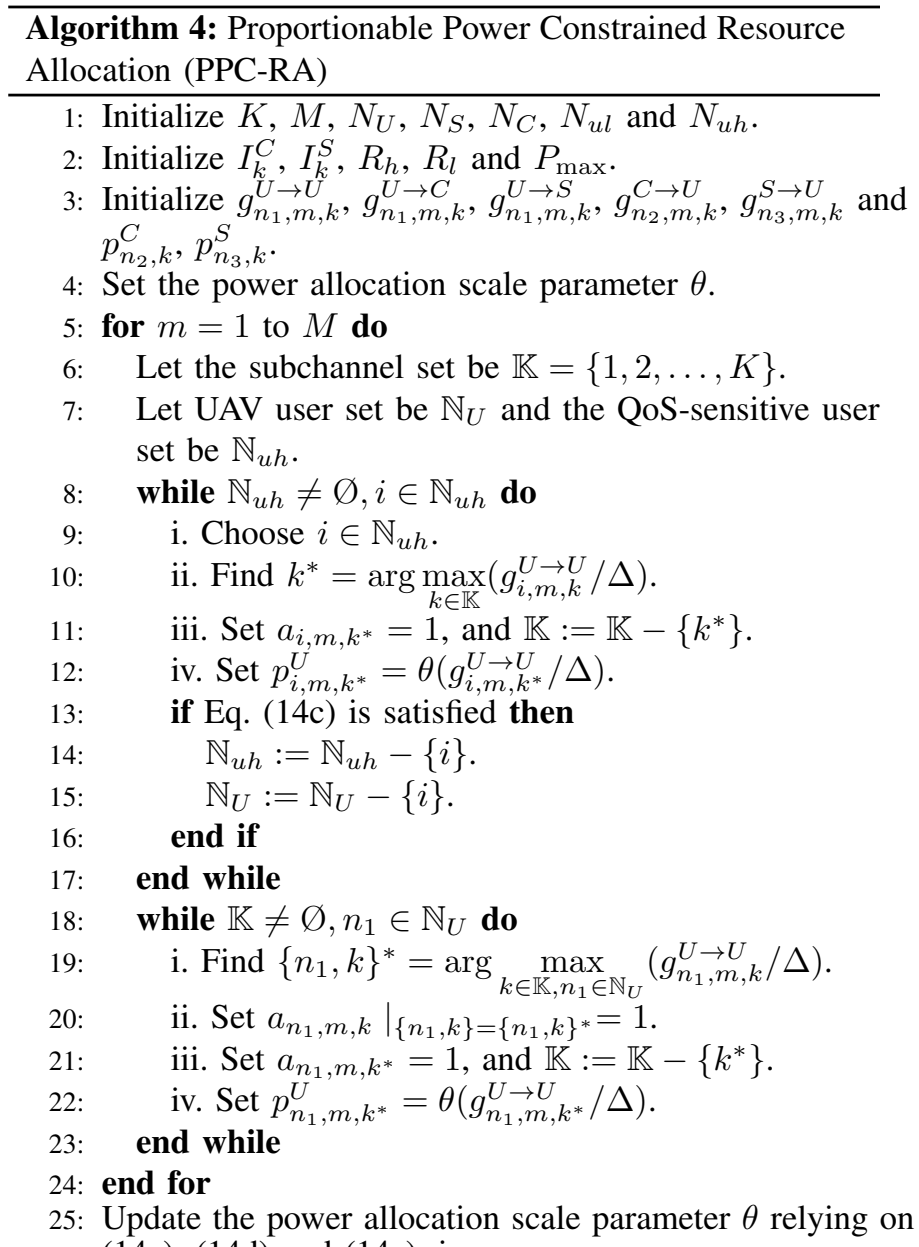
(14a), (14d) and (14e), i.e.

$$
\left.\begin{array}{rl}
\theta= & \min \left\{\frac{P_{\max }^{U} \Delta}{\sum_{k=1}^{K} a_{n_{1}, m, k} g_{n_{1}, m, k}^{U \rightarrow U}},\right. \\
& \frac{I_{k}^{C} \Delta}{\sum_{m=1}^{M} \sum_{n_{1}=1}^{N_{U}} a_{n_{1}, m, k} g_{n_{1}, m, k}^{U \rightarrow C} g_{n_{1}, m, k}^{U \rightarrow U}} \\
& \sum_{m=1}^{M} \sum_{n_{1}=1}^{N_{U}} a_{n_{1}, m, k} g_{n_{1}, m, k}^{U \rightarrow S} g_{n_{1}, m, k}^{U \rightarrow U}
\end{array}\right\} .
$$

26: Update the hovering altitude $\mathbf{h}$ of $M$ UAVs relying on Algorithm 1.

27: Denote $C_{\text {total }}^{*} \triangleq C_{\text {total }}\left(a_{n_{1}, m, k}, p_{n_{1}, m, k}^{U}, \mathbf{h}\right)$.

\section{B. Supplementary Analysis}

In this subsection, we provide a further explanation for our proposed two-stage resource allocation scheme in the face of both the optimization algorithm as well as the practical system design. As for solving the optimal power control problem in Eq. (24), the transmission power of both QoS-sensitive users and QoS-tolerant users is related to $\Delta / g_{n_{1}, m, k}^{U \rightarrow U}$ and $\Theta_{n_{1}}$, where $\Delta / g_{n_{1}, m, k}^{U \rightarrow U}$ represents the interference level from outside UAV networks, while $\Theta_{n_{1}}$ measures the interference 
level that the UAV users impose to other networks. We can conclude that the large $\Delta / g_{n_{1}, m, k}^{U \rightarrow U}$ may result in less power assigned to subchannel $k$. Furthermore, $\Theta_{n_{1}}$ also limits the power allocation for the sake of reducing influence to other communication systems. In contrast to the power allocation for QoS-tolerant users, $\mu_{n_{1}, m}$ tends to allocate more power for QoS-sensitive users, which yields a high data transmission rate. As for the subchannel allocation based on Eq. (28), due to the Lagrangian multiplier $\mu_{n_{1}, m}$ of Eq. (26), more subchannel resources may be assigned to the QoS-sensitive users. Hence, in practical system design, we should to a large degree use the subchannels having less interference from outside UAV networks and also imposing less interference to other networks, and allocate more such 'clean' subchannels and more power to QoS-sensitive users.

Moreover, the optimal hovering altitude of $M$ drones can be obtained by solving a series of reduced convex problems with the aid of CCP algorithm. According to Eq. (47) and Eq. (48), the optimal hovering altitude must be the boundary value of feasible region. To elaborate, $\sum_{i=1, i \neq m}^{M} h_{i}-(M-1) h_{m}$ in Eq. (47) measures the difference between the hovering altitude of the $m$-th UAV and the average altitude of others. Our algorithm aims to enlarge the gap between the hovering altitude of the $m$-th UAV and the average altitude of others.

In terms of the computational complexity, Algorithm 3 combines $j_{\max }$ iterations of the update of power control as well as the update of hovering altitude. Specifically, the computational complexity of the update of power control is $O\left(i_{\max } K M N_{U}\right)$, while the computational complexity of the update of hovering altitude $O\left(n_{\max } M\right)$, where $i_{\max }$ and $n_{\max }$ are the maximum number of iteration for each step, respective1y. Therefore, Algorithm 3 has a computational complexity of $O\left(j_{\max }\left(i_{\max } K M N_{U}+n_{\max } M\right)\right)$. By contrast, Algorithm 4 is a low-complexity greedy scheme aiming to preferentially satisfy the QoS-sensitive users, which gets rid of the update of a range of Lagrange dual. It has a much lower computational complexity of $O\left(M\left(N_{U}^{2}+\left(K-N_{U}\right)^{2}\right)+n_{\max } M\right)$ compared with Algorithm 3.

\section{Simulation Results}

In our simulation, three kinds of users are located in a $500 \mathrm{~m} \times 500 \mathrm{~m}$ square region. $N_{C}=10$ macrocell users and $N_{S}=10$ satellite users are randomly distributed in the area. Moreover, the coverage radius of each drone is $50 \mathrm{~m}$ and UAV users are randomly distributed in each coverage area. The altitude of GEO is $36000 \mathrm{~km}$ and only one MBS is considered in the simulation. The carrier frequency is $2.4 \mathrm{GHz}$ and the total number of the subchannel is $K=128$, each of which has a bandwidth of $15 \mathrm{kHz}$. The AWGN power spectrum density is $-174 \mathrm{dBm} / \mathrm{Hz}$. Furthermore, the channel between users and the MBS follows Rayleigh fading. By contrast, the channels between users and UAVs and the GEO follow Rician fading with $5 \mathrm{~dB}$ Rician factor. Let the reference-distance unit power gain be $\kappa=1.4 \times 10^{-4}$ [44]. The hovering altitude of drones spans from $200 \mathrm{~m}$ to $400 \mathrm{~m}$.

In the following, we consider two scenarios with 4 UAVs and 9 UAVs, respectively. Each drone serves $N_{U}=4$ UAV

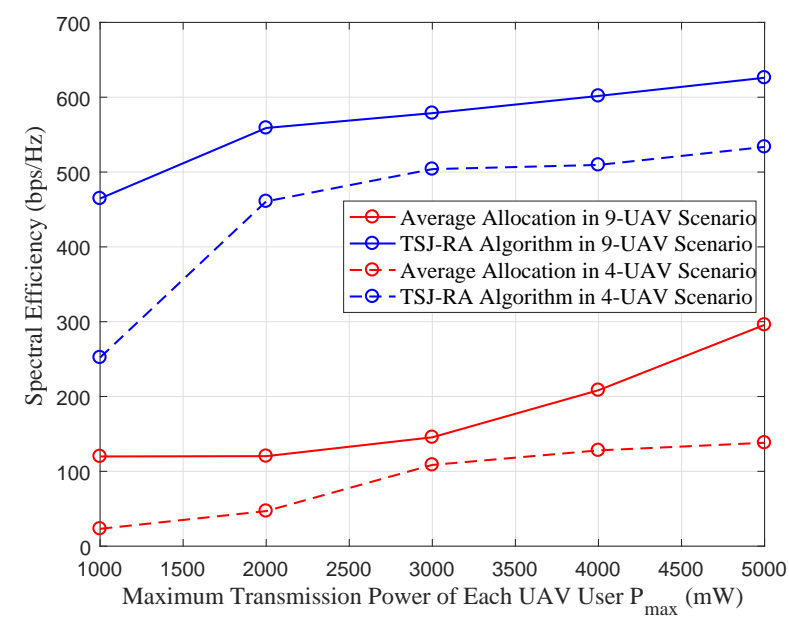

Fig. 2. Spectrum efficiency versus maximum transmission power of UAV users parameterized by different number of UAV networks.

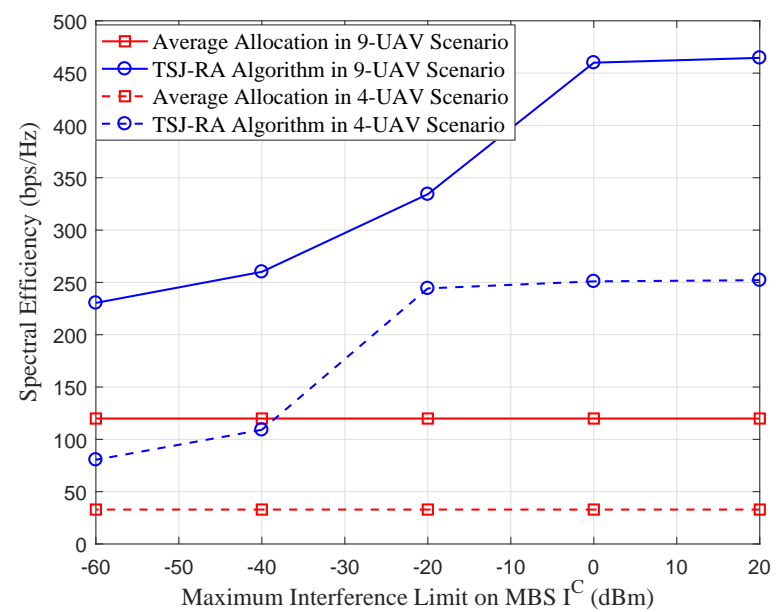

Fig. 3. Spectrum efficiency versus maximum interference limit of the MBS parameterized by different number of UAV networks.

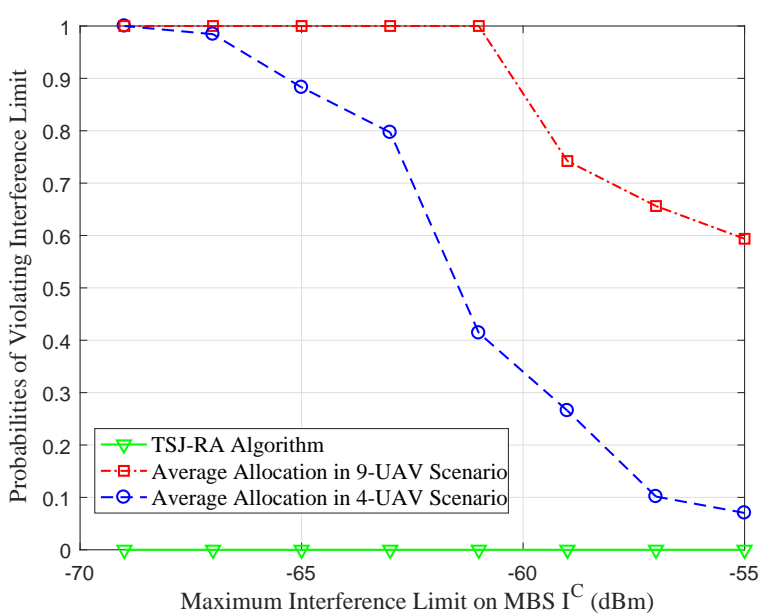

Fig. 4. Probabilities of violating the maximum interference limit on the MBS. 


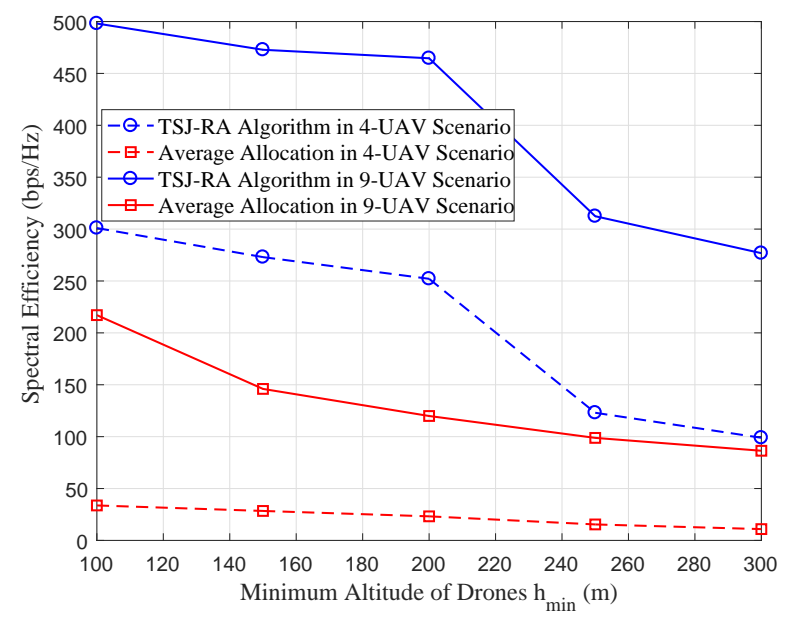

Fig. 5. Spectrum efficiency versus minimum hovering altitude parameterized by different number of UAV networks.

users. In the 4-UAV scenario, there are total 8 QoS-sensitive users and 8 QoS-tolerant users, while there are total 24 QoS-sensitive users and 12 QoS-tolerant users in the 9-UAV scenario. Moreover, the minimum data rate requirement of QoS-sensitive users is $R_{h}=30 \mathrm{kbps}$. We define the spectrum efficiency (SE) of UAV networks to evaluate the effectiveness of our proposed algorithm as: $\mathrm{SE}=C_{\text {total }} / B \quad(\mathrm{bps} / \mathrm{Hz})$.

Fig. 2 shows the impact of the maximum transmission power $p_{\max }^{U}$ on the UAV network's SE, where the maximum interference limit of both the MBS and the GEO is $0 \mathrm{dBm}$, i.e. $I^{C}=0 \mathrm{dBm}$ and $I^{S}=0 \mathrm{dBm}$ for all subchannels $k \in\{1,2, \ldots, K\}$. It can be observed that our proposed TSJ-RA algorithm outperforms the average resource allocation scheme $^{2}$ in terms of the SE. It is because the proposed TSJRA algorithm jointly optimizes the altitudes of the drones and transmission power of all users, achieving a decent SE performance and satisfying all the constraints all the time. As a comparison, the comparison algorithm is not aware of system configuration and introduces significant SE loss. Besides, higher SE is obtained with a loose transmission power constraint. Meanwhile, a dense UAV deployment is capable of substantially increasing the network's SE.

Fig. 3 demonstrates the performance of UAV network's SE characterized by the maximum interference limit of the MBS, i.e. $I^{C}$, with respect to $P_{\max }^{U}=1000 \mathrm{~mW}$ and $I^{S}=0 \mathrm{dBm}$. Since the average resource allocation scheme does not rely on the interference limit, the spectrum efficiency is not improved with the increase of MBS's interference limit. As for the TSJRA algorithm, a loose interference limit on the MBS yields a high SE of UAV networks to some extent. It is because that with a loose interference limit, UAV users are capable of using higher transmission power, while with a strict interference limit, UAV users have to properly decrease the transmission power to satisfy the preset constraint. Besides, it can seen that when

\footnotetext{
${ }^{2}$ In this paper, the average resource allocation scheme means that subchannels as well as power are uniformly allocated to two kinks of users without considering the interference limit of the MBS and the GEO under the constraint of a secure hovering altitude of each drone.
}

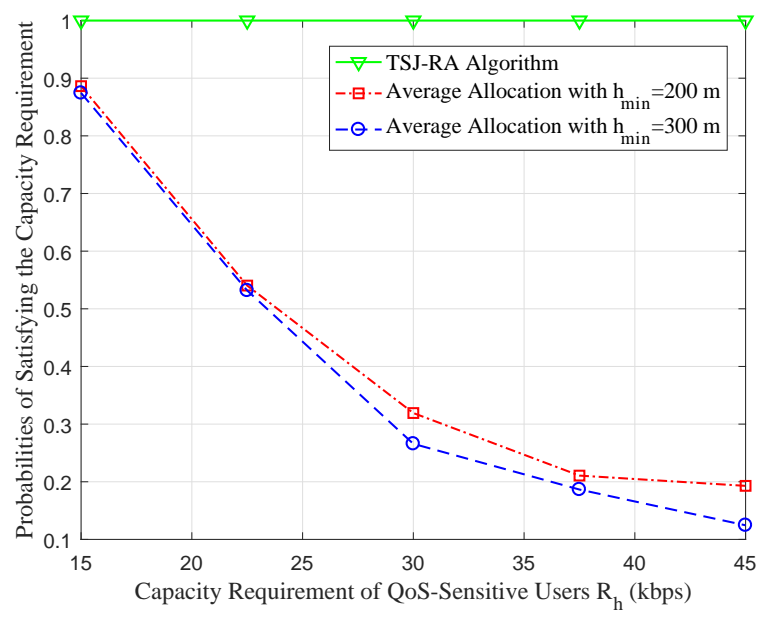

Fig. 6. Probabilities of satisfying capacity requirement of QoS-sensitive users in terms of different minimum hovering altitude of drones.

the interference limit is loose enough, such as $I^{C}=0 \mathrm{dBm}$ for 9-UAV scenario and $I^{C}=-20 \mathrm{dBm}$ for 4 -UAV scenario, the $\mathrm{SE}$ remains unchanged. It is because with a loose threshold, the pre-set interference constraint can be always satisfied with the given maximum available transmission power. To elaborate a little further, Fig. 4 portrays the probabilities of violating the maximum interference limit on the MBS, which is defined as the ratio of the number of subchannels with interference higher than pre-set maximum limit to the total number of subchannels. We can conclude that our proposed algorithm satisfy the interference limit for all subchannels $k \in\{1,2, \ldots, K\}$ at all given $I^{C}$ values. However, the average algorithm has a high probability of violating the interference limit when the interference requirement is stringent.

In Fig. 5, we evaluate the performance of SE versus different minimum hovering altitudes in different scenarios. It can be seen that a lower minimum hovering altitude is beneficial in terms of improving the SE of the total UAV networks relying on both our proposed TSJ-RA algorithm as well as on the average algorithm. Furthermore, Fig. 6 demonstrates the probabilities of satisfying the pre-set capacity requirement for QoS-sensitive users versus different values of $R_{h}$, which is defined as the ratio of the number of QoSsensitive users with satisfied capacity to the total number of QoS-sensitive users. It can be seen that our proposed algorithm always outperforms the comparison algorithm at all given $R_{h}$ values. It is because that our the proposed algorithm considers the capacity requirement of QoS-sensitive users, making the QoS-sensitive users have high priorities to obtain the channels. As a result, the probability of satisfying capacity requirement for QoS-sensitive users equals to 1 all the time. By contrast, the average allocation is not aware of the pre-set capacity constraint. Especially when the capacity requirement is stringent, i.e. $40 \mathrm{kbps}$, only around $10 \%$ of QoS-sensitive users can achieve decent capacity higher than the pre-set constraint. 


\section{CONCLUSIONS}

In this paper, we formulated a two-stage joint hovering altitude and power control for UAV networks considering the feasible deployment of drones in the context of a space-airground three-tier heterogeneous network for supporting IoT applications. After appropriate convex relaxation, we used Lagrange dual decomposition and CCP method to provide a near optimal solution for our proposed problem, followed by a low-complexity proportionable power constrained resource allocation algorithm. Finally, extensive simulations were conducted in order to show the performance of our resource allocation mechanism, which yielded an improved UAV network's throughput.

\section{REFERENCES}

[1] L. Gupta, R. Jain, and G. Vaszkun, "Survey of important issues in UAV communication networks," IEEE Communications Surveys \& Tutorials, vol. 18, no. 2, pp. 1123-1152, Nov. 2016.

[2] J. Wang, C. Jiang, Z. Han, Y. Ren, R. G. Maunder, and L. Hanzo, "Taking drones to the next level: Cooperative distributed unmannedaerial-vehicular networks for small and mini drones," IEEE VehIcular Technology MagazIne, vol. 12, no. 3, pp. 73-82, Sept. 2017.

[3] M. Mozaffari, W. Saad, M. Bennis, and M. Debbah, "Efficient deployment of multiple unmanned aerial vehicles for optimal wireless coverage," IEEE Communications Letters, vol. 20, no. 8, pp. 1647-1650, Aug. 2016.

[4] Q. Yu, J. Wang, and L. Bai, "Architecture and critical technologies of space information networks," Journal of Communications and Information Networks, vol. 1, no. 3, pp. 1-9.

[5] L. Bai, L. Zhu, X. Zhang, W. Zhang, and Q. Yu, "Multi-satellite relay transmission in 5G: Concepts, techniques and challenges," to be published in IEEE Network, Sep. 2018.

[6] M. Mozaffari, W. Saad, M. Bennis, Y.-H. Nam, and M. Debbah, "A tutorial on UAVs for wireless networks: Applications, challenges, and open problems," arXiv preprint arXiv:1803.00680, 2018.

[7] M. De Sanctis, E. Cianca, G. Araniti, I. Bisio, and R. Prasad, "Satellite communications supporting Internet of remote things," IEEE Internet of Things Journal, vol. 3, no. 1, pp. 113-123, Feb. 2016.

[8] Y. Zhou, N. Cheng, N. Lu, and X. S. Shen, "Multi-UAV-aided networks: aerial-ground cooperative vehicular networking architecture," IEEE Vehicular Technology Magazine, vol. 10, no. 4, pp. 36-44, Dec. 2015.

[9] A. Merwaday and I. Guvenc, "UAV assisted heterogeneous networks for public safety communications," in IEEE Wireless Communications and Networking Conference Workshops (WCNCW), New Orleans, LA, Mar. 2015, pp. 329-334

[10] Y. Zeng, R. Zhang, and T. J. Lim, "Wireless communications with unmanned aerial vehicles: opportunities and challenges," IEEE Communications Magazine, vol. 54, no. 5, pp. 36-42, May 2016.

[11] R. Amorim, H. Nguyen, P. Mogensen, I. Kovacs, J. Wigard, and T. Sorensen, "Radio channel modelling for UAV communication over cellular networks," IEEE Wireless Communications Letters, vol. 6, no. 4, pp. 514-517, Aug. 2017.

[12] Y. Zeng and R. Zhang, "Energy-efficient UAV communication with trajectory optimization," IEEE Transactions on Wireless Communications, vol. 16, no. 6, pp. 3747-3760, Jun. 2017.

[13] Z. M. Fadlullah, D. Takaishi, H. Nishiyama, N. Kato, and R. Miura, "A dynamic trajectory control algorithm for improving the communication throughput and delay in UAV-aided networks," IEEE Network, vol. 30, no. 1, pp. 100-105, Jan. 2016.

[14] M. Mozaffari, W. Saad, M. Bennis, and M. Debbah, "Unmanned aerial vehicle with underlaid device-to-device communications: Performance and tradeoffs," IEEE Transactions on Wireless Communications, vol. 15, no. 6 , pp. 3949-3963, Jun. 2016.

[15] B. Evans, M. Werner, E. Lutz, M. Bousquet, G. E. Corazza, G. Maral, and R. Rumeau, "Integration of satellite and terrestrial systems in future multimedia communications," IEEE Wireless Communications, vol. 12, no. 5 , pp. $72-80$, Oct.

[16] R. Suffritti, G. E. Corazza, A. Guidotti, V. Petrini, D. Tarchi, A. VanelliCoralli, and M. Di Renzo, "Cognitive hybrid satellite-terrestrial systems," in ACM 4th International Conference on Cognitive Radio and Advanced Spectrum Management, Barcelona, Spain, Oct. 2011, p. 64.
[17] E. Lagunas, S. K. Sharma, S. Maleki, S. Chatzinotas, and B. Ottersten, "Resource allocation for cognitive satellite communications with incumbent terrestrial networks," IEEE Transactions on Cognitive Communications and Networking, vol. 1, no. 3, pp. 305-317.

[18] J. Wang, C. Jiang, Z. Han, Y. Ren, and L. Hanzo, "Network association strategies for an energy harvesting aided super-WiFi network relying on measured solar activity." IEEE Journal on Selected Areas in Communications, vol. 34, no. 12, pp. 3785-3797, Dec. 2016.

[19] Q. Yu, C. Han, L. Bai, C. Jinbo, and X. S. Shen, "Low-complexity multiuser detection in millimeter-wave systems based on opportunistic hybrid beamforming," IEEE Transactions on Vehicular Technolog, DOI: 10.1109/TVT.2018.2864615, pp. 1-5, Aug. 2018.

[20] N. Rupasinghe, Y. Yapici, I. Güvenç, and Y. Kakishima, "Nonorthogonal multiple access for mmWave drones with multi-antenna transmission," in The 51st Asilomar Conference on Signals, Systems, and Computers, Pacific Grove, CA, Oct. 2017, pp. 958-963.

[21] 3GPP, "Study on new radio (NR) to support non-terrestrial networks," TR 38.811 .

[22] Z. Xiao, P. Xia, and X.-G. Xia, "Enabling UAV cellular with millimeterwave communication: Potentials and approaches," IEEE Communications Magazine, vol. 54, no. 5, pp. 66-73, May 2016.

[23] Z. Xiao, L. Zhu, J. Choi, P. Xia, and X.-G. Xia, "Joint power allocation and beamforming for non-orthogonal multiple access (NOMA) in 5G millimeter-wave communications," IEEE Transactions on Wireless Communications, vol. 17, no. 5, pp. 2961-2974, May 2018.

[24] A. Y.-M. Lin, A. Novo, S. Har-Noy, N. D. Ricklin, and K. Stamatiou, "Combining GeoEye-1 satellite remote sensing, UAV aerial imaging, and geophysical surveys in anomaly detection applied to archaeology," IEEE Journal of Selected Topics in Applied Earth Observations and Remote Sensing, vol. 4, no. 4, pp. 870-876, Dec. 2011.

[25] V. Sharma, M. Bennis, and R. Kumar, "UAV-assisted heterogeneous networks for capacity enhancement," IEEE Communications Letters, vol. 20 , no. 6, pp. 1207-1210, Jun. 2016.

[26] J. Zhang, S. Chen, R. G. Maunder, R. Zhang, and L. Hanzo, "Adaptive coding and modulation for large-scale antenna array based aeronautical communications in the presence of cochannel interference," IEEE Transactions on Wireless Communications, DOI:10.1109/TWC.2017.2777985, Dec. 2017.

[27] D. Fooladivanda and C. Rosenberg, "Joint resource allocation and user association for heterogeneous wireless cellular networks," IEEE Transactions on Wireless Communications, vol. 12, no. 1, pp. 248-257.

[28] Y. Choi, H. Kim, S.-w. Han, and Y. Han, "Joint resource allocation for parallel multi-radio access in heterogeneous wireless networks," IEEE Transactions on Wireless Communications, vol. 9, no. 11, pp. 33243329, Nov. 2010

[29] R. Xie, F. R. Yu, H. Ji, and Y. Li, "Energy-efficient resource allocation for heterogeneous cognitive radio networks with femtocells," IEEE Transactions on Wireless Communications, vol. 11, no. 11, pp. 3910 3920, Nov. 2012.

[30] Q. Ye, B. Rong, Y. Chen, M. Al-Shalash, C. Caramanis, and J. G. Andrews, "User association for load balancing in heterogeneous cellular networks," IEEE Transactions on Wireless Communications, vol. 12, no. 6, pp. 2706-2716, Jun. 2013.

[31] H. Zhang, C. Jiang, N. C. Beaulieu, X. Chu, X. Wen, and M. Tao, "Resource allocation in spectrum-sharing OFDMA femtocells with heterogeneous services," IEEE Transactions on Communications, vol. 62, no. 7, pp. 2366-2377, Jul. 2014.

[32] H. Wang, J. Wang, G. Ding, L. Wang, T. A. Tsiftsis, and P. K. Sharma, "Resource allocation for energy harvesting-powered D2D communication underlaying UAV-assisted networks," IEEE Transactions on Green Communications and Networking, Oct. 2017.

[33] C. E. Shannon, "A mathematical theory of communication," $A C M$ SIGMOBILE Mobile Computing and Communications Review, vol. 5, no. 1, pp. 3-55, Jan. 2001.

[34] D. Zhu, J. Wang, A. L. Swindlehurst, and C. Zhao, "Downlink resource reuse for device-to-device communications underlaying cellular networks," IEEE Signal Processing Letters, vol. 21, no. 5, pp. 531-534, May 2014.

[35] R. G. Stephen and R. Zhang, "Joint millimeter-wave fronthaul and OFDMA resource allocation in ultra-dense CRAN," IEEE Transactions on Communications, vol. 65, no. 3, pp. 1411-1423, Jan. 2017.

[36] C. Y. Wong, R. S. Cheng, K. B. Lataief, and R. D. Murch, "Multiuser OFDM with adaptive subcarrier, bit, and power allocation," IEEE Journal on selected areas in communications, vol. 17, no. 10, pp. 17471758, Oct. 1999. 
[37] K. Seong, M. Mohseni, and J. M. Cioffi, "Optimal resource allocation for OFDMA downlink systems," in IEEE International Symposium on Information Theory, Seattle, WA, Jul. 2006, pp. 1394-1398.

[38] S. Boyd and L. Vandenberghe, Convex optimization. Cambridge university press, 2004.

[39] M. Held, P. Wolfe, and H. P. Crowder, "Validation of subgradient optimization," Mathematical Programming, vol. 6, no. 1, pp. 62-88, Dec. 1974.

[40] M. L. Fisher, "The Lagrangian relaxation method for solving integer programming problems," Management Science, vol. 27, no. 1, pp. 1-18, Jan. 1981.

[41] D. P. Palomar and M. Chiang, "A tutorial on decomposition methods for network utility maximization," IEEE Journal on Selected Areas in Communications, vol. 24, no. 8, pp. 1439-1451, Aug. 2006.

[42] X. Shen, S. Diamond, Y. Gu, and S. Boyd, "Disciplined convex-concave programming," in IEEE 55th Conference on Decision and Control (CDC), Las Vegas, NV, Dec. 2016, pp. 1009-1014.

[43] T. Lipp and S. Boyd, "Variations and extension of the convex-concave procedure," Optimization and Engineering, vol. 17, no. 2, pp. 263-287, Jun. 2016.

[44] H. He, S. Zhang, Y. Zeng, and R. Zhang, "Joint altitude and beamwidth optimization for UAV-enabled multiuser communications," IEEE COmmunications Letters, DOI:10.1109/LCOMM.2017.2772254, Nov. 2017.

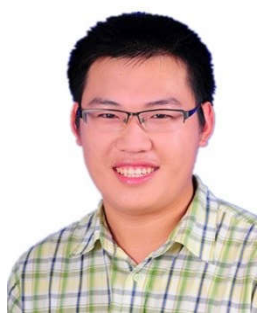

Jingjing Wang (S'14) received his B.S. degree in Electronic Information Engineering from Dalian University of Technology in 2014 with the highest honor. He currently works for his $\mathrm{PhD}$ degree in Department of Electronic Engineering, Tsinghua University, Beijing. From 2017 to 2018, he has been a joint $\mathrm{PhD}$ student in Next Generation Wireless Group chaired by Prof. Lajos Hanzo, University of Southampton, UK. His research interests include the resource allocation and network association, learning theory aided modeling, analysis and signal processing, as well as information diffusion theory for mobile wireless networks. $\mathrm{He}$ received Tsinghua GuangHua Scholarship Award in 2016 and graduate China National Scholarship Award in 2017.

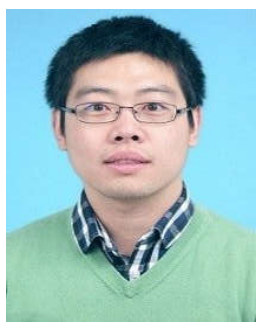

Chunxiao Jiang (S'09-M'13-SM'15) received his B.S. degree in information engineering from Beijing University of Aeronautics and Astronautics (Beihang University) in 2008 and the Ph.D. degree from Tsinghua University, Beijing in 2013, both with the highest honors. During 2011-2012, he visited the Signals and Information Group at Department of Electrical \& Computer Engineering of University of Maryland. During 2013-2016, he was a postdoc researcher at Department of Electronic Engineering, Tsinghua University. Dr. Jiang is currently an assistant research fellow at Tsinghua Space Center. His research interests include the applications of game theory and queuing theory in wireless communication and networking. Dr. Jiang received Best Paper Award from IEEE GLOBECOM in 2013, Best Student Paper Award from IEEE GlobalSIP in 2015, Tsinghua Outstanding Postdoc Award in 2015, Beijing Distinguished Graduated Student Award, Chinese National Fellowship and Tsinghua Outstanding Distinguished Doctoral Dissertation in 2013.

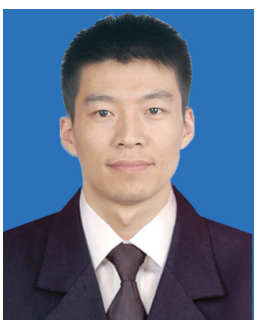

Zhongxiang Wei (S'15CM'17) received the Ph.D. degree in electrical engineering and electronics from the University of Liverpool, Liverpool, U.K, in 2017. From March 2016 to March 2017, he was with the Institution for Infocomm Research, Agency for Science, Technology, and Research, Singapore, as a research assistant. From March 2017 to October 2017, he was a visiting student with the Wireless Networks and Communications Group, Harbin Institute of Technology, Shenzhen, China. Since early 2018, he has been working with the University College London (UCL), London, as a research associate. His research interests include constructive interference design, green communications, full-duplex, millimeter-wave communications and algorithm design. He was the recipient of the Graduate China National Scholarship Award in 2012, the recipient of the A*STAR Research Attachment Programme (ARAP) Studentship in 2016 and the recipient of the CSC Outstanding Self-Financed Scholarship in 2017.

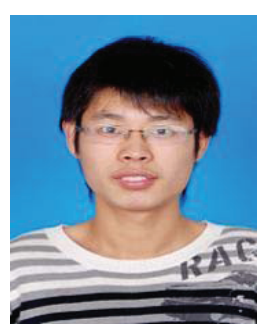

Cunhua Pan received his B.S. and Ph.D. degrees in school of Information Science and Engineering, Southeast University, Nanjing, China, in 2010 and 2015 , respectively. From 2015 to 2016, he worked as a research associate in University of Kent, UK. He is currently a Postdoc with Queen Mary University of London, UK. His research interests mainly include Ultra-dense C-RAN, machine learning, UAV, internet of things (IoT), and mobile edge computing. He serves as Editor for IEEE ACCESS, Student Travel Grant Chair for ICC 2019, and TPC Member for many conferences, such as ICC and Globecom.

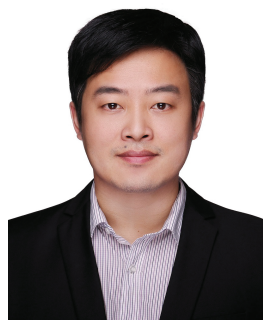

Haijun Zhang (M'13-SM'17) is currently a Full Professor in University of Science and Technology Beijing, China. He was a Postdoctoral Research Fellow in Department of Electrical and Computer Engineering, the University of British Columbia (UBC), Vancouver Campus, Canada. He serves as Editor of IEEE Transactions on Communications, IEEE Transactions on Green Communications and Networking, IEEE 5G Tech Focus, and serves/served as a Leading Guest Editor for IEEE Communications Magazine, and IEEE Transactions on Emerging Topics in Computing. He serves/served as General Co-Chair of GameNets'16, Symposium Chair of Globecom'19, TPC Co-Chair of INFOCOM'18 Workshop IECCO, General Co-Chair of ICC'18/ICC'17/Globecom'17 Workshop on UDN, and General Co-Chair of Globecom'17 Workshop on LTE-U. He received the IEEE CSIM Technical Committee Best Journal Paper Award in 2018 and IEEE ComSoc Young Author Best Paper Award in 2017.

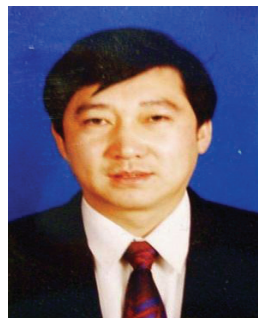

Yong Ren (SM'16) received his B.S, M.S and $\mathrm{Ph} . \mathrm{D}$. degrees in electronic engineering from Harbin Institute of Technology, China, in 1984, 1987, and 1994, respectively. He worked as a post doctor at Department of Electronics Engineering, Tsinghua University, China from 1995 to 1997. Now he is a professor of Department of Electronics Engineering and the director of the Complexity Engineered Systems Lab in Tsinghua University. He holds 12 patents, and has authored or co-authored more than 100 technical papers in the behavior of computer network, P2P network and cognitive networks. He has serves as a reviewer of IEICE Transactions on Communications, Digital Signal Processing, Chinese Physics Letters, Chinese Journal of Electronics, Chinese Journal of Computer Science and Technology, Chinese Journal of Aeronautics and so on. His current research interests include complex systems theory and its applications to the optimization and information sharing of the Internet, Internet of Things and ubiquitous network, cognitive networks and Cyber-Physical Systems. 